\title{
Multi-Level Modeling Methodology for Optimal Design of Electric Machines Based on Multi-Disciplinary Design Optimization
}

\author{
Zehua Dai ${ }^{1}$, Li Wang ${ }^{1} * \mathbb{C}^{\mathbb{D}}$, Lexuan Meng ${ }^{2} \mathbb{D}$, Shanshui Yang ${ }^{1}$ and Ling Mao ${ }^{1}$ \\ 1 Collage of Automation, Nanjing University of Aeronautics and Astronautics, Nanjing 210000, China; \\ dzh525800605@nuaa.edu.cn (Z.D.); yshanshui@nuaa.edu.cn (S.Y.); maudymao@nuaa.edu.cn (L.M.) \\ 2 AC Systems, Power Grid division, ABB, 8000 Zurich, Sweden; lexuan.meng@gmail.com \\ * Correspondence: liwang@nuaa.edu.cn; Tel.: +86-138-5163-2296
}

Received: 6 September 2019; Accepted: 29 October 2019; Published: 1 November 2019

\begin{abstract}
The transportation sector is undergoing electrification to gain advantages such as lighter weight, improved reliability, and enhanced efficiency. As contributors to the safety of embedded critical functions in electrified systems, better sizing of electric machines in vehicles is required to reduce the cost, volume, and weight. Although the designs of machines are widely investigated, existing studies are mostly complicated and application-specific. To satisfy the multi-level design requirements of power systems, this study aims to develop an efficient modeling method of electric machines with a background of aircraft applications. A variable-speed variable-frequency (VSVF) electrically excited synchronous generator is selected as a case study to illustrate the modular multi-physics modeling process, in which weight and power loss are the major optimization goals. In addition, multi-disciplinary design optimization (MDO) methods are introduced to facilitate the optimal variable selection and simplified model establishment, which can be used for the system-level overall design. Several cases with industrial data are analyzed to demonstrate the effectiveness and superior performance of the modeling method. The results show that the proposed practices provide designers with accurate, fast, and systematic means to develop models for the efficient design of aircraft power systems.
\end{abstract}

Keywords: electrified aircraft; electric machine; MDO; intelligent design; system-level design; statistical learning; surrogate model

\section{Introduction}

Electrified transportation reveals significant benefits in terms of increased reliability, improved energy efficiency, reduced emissions, and enhanced passenger comfort. With the breakthrough of power electronics, the adoption of the More Electric Aircraft (MEA) and electric propulsion aircraft concepts have become a major trend in the air industry [1,2]. As a result, many hydraulic and pneumatic power-driven systems are being replaced by their electrical counterparts in existing MEAs, e.g., Boeing 787 [3] and Airbus 380 [4]. Moreover, the hybrid/all-electric propulsion electric grid is now being investigated [5-7]. In electrified aircraft, the electric machine is the key equipment to realize the conversion and transformation of multi-physical energies; therefore, the sizing of the machine has become one of the leading topics in the study of transport electrification.

In addition, with the development of artificial intelligence and information technologies, model-based system engineering (MBSE) [8-10] and optimization-assisted design [11] have become the focus of researchers, becoming increasingly mature, and will be particularly powerful for the design of aircraft power systems. In this context, models of the electrical environment control system (ECS) [12,13], 
electromagnetic actuators (EMAs) [14-17], and electro-hydraulic actuators (EHAs) $[16,18,19]$ were established to support the trade-offs between the weight and power loss of More-electric systems. To implement these analyses, machine models for optimal designs have been widely investigated. The magnetic equivalent circuit (MEC) model has been used in the electromagnetic design of machines, given the multiple flux barrier and the variables (permeance, potential, etc.). However, the model requires external structural and thermal designs to gain better performance [20-24]. The finite element model (FEM) can illustrate comprehensive characteristics of multi-physical fields of machines accurately. Nevertheless, it is highly complicated and computationally expensive to extract performance indexes, leading to difficult trade-offs. Therefore, it has mainly been applied to the detailed design phase of machines [25-27]. Multi-physics models present the electromagnetic, geometrical, and thermal characteristics so that couplings of multi-physics can be evaluated to help assess the More-electric degree of aircraft [13]. However, the multi-physics model of the permanent magnet synchronous generator (PMSG) developed in [20] has 238 inputs and 941 outputs, which indicates that it results in high computational costs, and is not suitable for system-level studies [28]. In contrast, the multi-physics model in [29] has relatively low complexity. However, only the iron loss was considered the model, and couplings among components were not explicitly defined. In addition, although the multi-physics model has been simplified based on physical energetical equivalence, the eight-dimensional design space leads to the limited application to studies of More-electric systems [13]. The use of scaling law (SL) has the advantage of requiring only a reference machine for a complete estimation of a product range, which provides the designer with parameters needed for integration [30]. However, the method is project-specific and ignores the multi-physics couplings of machines. On the other hand, the law can be broken due to some limits, e.g., with an increase of power, a cooling method may be required to satisfy the permissible temperature rise. In power grid applications, machine models are usually built as polynomial functions of power, illustrating the influences of the energy allocation on the cost at a system level. Nevertheless, they are statistical results of particular products, and not yet applicable for aircraft applications [31].

In summary, although the sizing of machines is widely investigated, an efficient modeling system of machines for the design of aircraft power systems is still lacking. To meet the demand for aircraft electrification, a machine model for the design should satisfy both the descriptions of multi-physics couplings and ease of use [32], which requires a trade-off between accuracy and speed.

In this context, multi-disciplinary design optimization (MDO) allows for contending with the strong couplings of high-complex design problems by applying technologies of model approximation, sensitivity analysis, system problem decomposition, and coordination strategies, which inspires our work [33]. Moreover, MDO applications in power systems have been proved to be attractive in the literature. The design problem of the ECS is decomposed into the optimization of different subsystems, e.g., the filter, inverter, and machine, by the collaborative optimization method in [13]. According to different disciplines, the design of the permanent magnet synchronous motor is divided into problems of structure, manufacture, and multi-physics [34]. However, the MDO method has not been applied to machine modeling in existing studies.

Accordingly, the main contribution of this study is to propose a novel model exchange process based on MDO to satisfy multi-level design requirements of electric machines in the aircraft power system, e.g., consistency, simplicity, and accuracy. The work is based on the improvement of the existing multi-physics model in [29].

The remainder of this paper is organized as follows. Section 2 presents the multi-level modeling method for the design and evaluation of machines in the aircraft power system. To illustrate the method, Section 3 develops a modular multi-physics model of machines based on the direct design approach [35] to help the component integration. Section 4 is dedicated to the exploration of the relationships and trends between inputs and outputs of the multi-physics model to identify key factors and to obtain the optimal parameter combination for system-level machine models. Different simplified machine models are then built and compared. Several cases are investigated and compared in Section 5 
to verify the promising performance of the proposed method. Finally, the features of the proposed model and probable applications in future studies are discussed in the conclusion.

\section{Modeling Method of Electric Machine for Design and Evaluation}

\subsection{Modeling Needs Analysis}

Traditional machine simulation models calculate variables of power and energy used for the component selections [14]. Both steady-state and dynamic simulations are mandatory due to the complex couplings of multi-physical characteristics. These simulations are usually based on lumped circuit models or field models, and run in environments such as MATLAB/Simulink, Dymola, and ANSOFT. To run this kind of model, it is necessary that all component parameters should be known. Meanwhile, it is highly time-consuming to extract features from models. Therefore, it is suitable for detailed design, analysis, and verification, whereas time-independent symbolic analytical models are preferred for assisting with system integration.

From the perspective of design optimization, design requirements can be translated into design, constraint, and target variables of different levels. Then, the design is conducted hierarchically by algebraic solvers or intelligent algorithms to achieve the best goals with the satisfaction of constraints by well-determined design variables; see Figure 1. As the impacts of variations of variables can be propagated both vertically (from bottom to top, e.g., components, machine, and system) and horizontally (e.g., among components), every design is a combinational optimization problem based on both the underlying design results and the synthesis forms of underlying-level designs, e.g., the selected component technologies and the selected machine structure in the machine design process. Therefore, iterative calculations may occur because of the improper design or integration of the underlying units; in Figure 1, see the red and green-colored directed lines. Therein, the green-colored lines can be avoided by optimization algorithms, whereas the red-colored lines cannot be avoided and may lead to blind designs, due to the invisible and complex cross-links among variables.

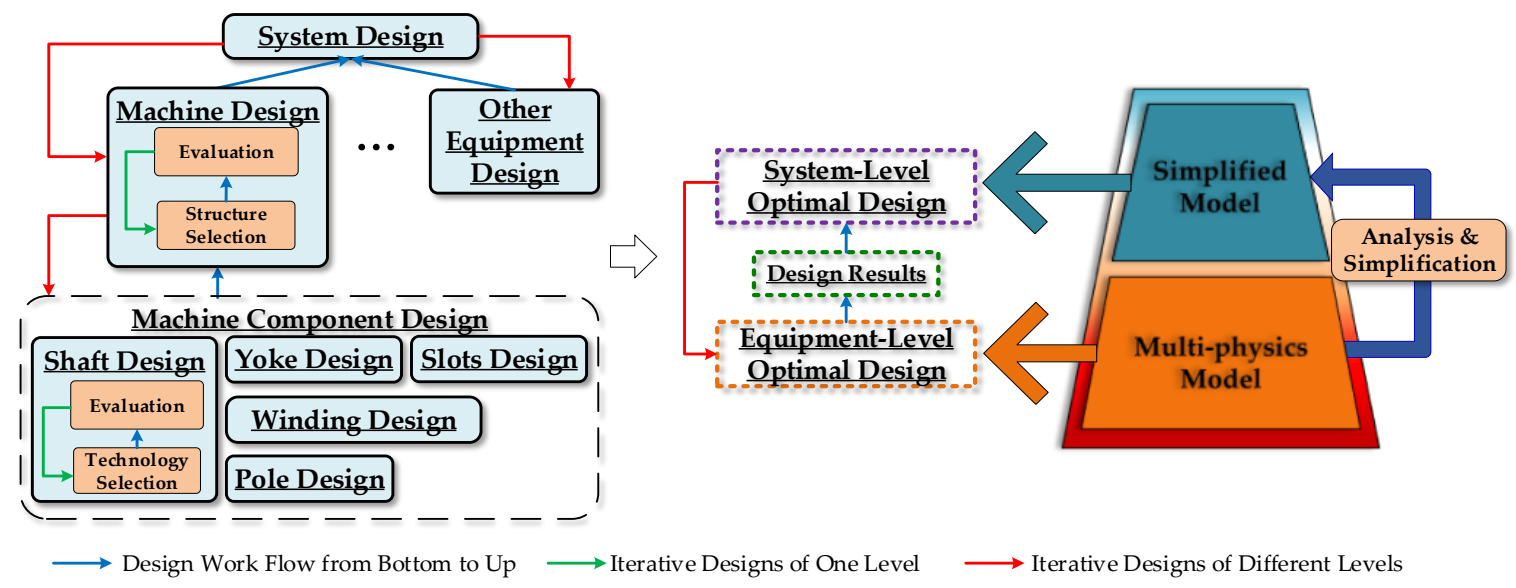

Figure 1. The hierarchical design process of a More-electric system and required evaluation models.

Accordingly, the hierarchical designs require a well-structured modeling approach:

- The primary-level model handles the design of the machine for the integration of More-electric systems, linking dimensions and characteristics of selected materials and technologies to multi-physical characteristics of components with full consideration of their energy conversion features [29]. Thus, couplings of variables can be illustrated clearly.

- The secondary-level model allows proper simplification of the primary-level multi-physics model, which focuses on the performance evaluation of the system, and should be consistent with the primary-level model. 
In this way, the developed modeling approach covers enough information needed for different levels of design and evaluation, and appears to be the most appropriate means of accelerating the design process, which is the major task and objective of this paper.

\subsection{Modular Multi-Physics Model}

To demonstrate the propagations of the impacts of variables, both multi-physical characteristics and combinatorial explosions of components are required in the multi-physics model. On the other hand, the modular approach in electrical machines denotes that their parts (the stator, the rotor, or both) are made up of distinctive segments, which can increase flexibility of design. Therefore, the structure of the improved multi-physics model is illustrated in Figure 2.

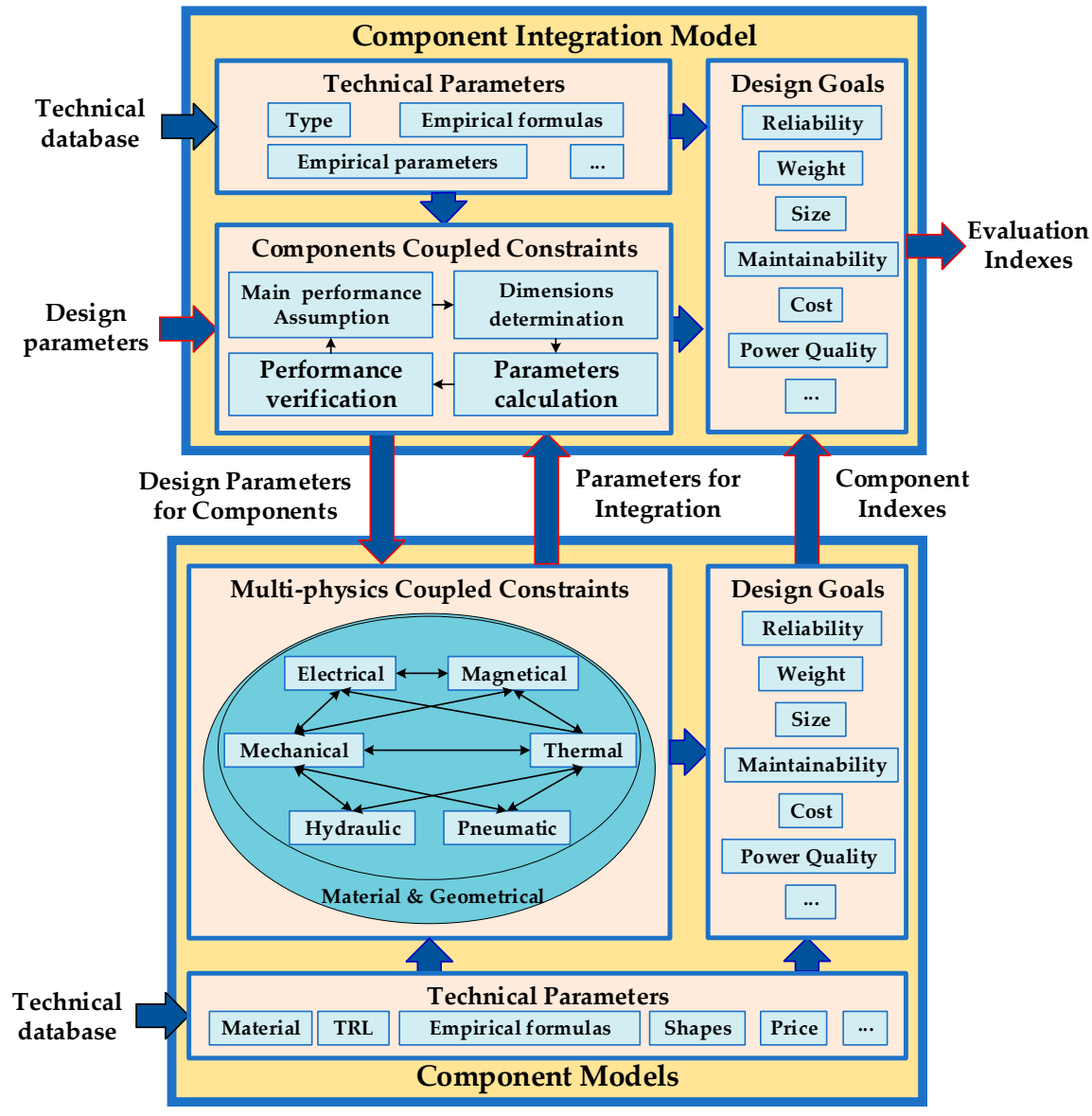

Figure 2. Multi-physics model of a machine.

Every component model comprises three parts, namely, technical parameters, multi-physics coupled constraints, and the design goal, in which the multi-physics coupled constraints represent the core linking the other two parts. Technical parameters comprise the design codes from design experiences, standards, and protocols (i.e., densities and strengths of materials, technology readiness level (TRL), etc.), which are used as pre-specified design requirements. According to the selected configurations in the technical parameters, the multi-physics coupled constraints describe the relationship between design variables and performance according to the principle of energy conversion and transmission; structure parameters describing the geometry and material information can be then obtained. Based on these two parts, the design goal calculates evaluation indexes depending on design requirements.

The component integration model has a similar structure to component models. The difference is in the components coupled constraints describing the cross-links of components. Differently from 
the traditional machine design method, the machine here is designed based on the direct approach. The detailed process is as follows: according to the required performance (i.e., the rated power, operating points of components, etc.), main radial and axial dimensions are initially calculated by the basic equations of the machine; then, detailed parameters of components (e.g., dimensions of the rotor, stator, yoke, or windings) are determined by optimal technology selections; next, the whole machine is integrated in aspects of structural, electromagnetic, and thermal features; finally, constraints and initial assumptions are checked to verify the design. Calculations in [35] show that the differences between initially assumed parameters and calculated results based on the direct approach are small enough to form the closed loop of design.

In this way, functional and performance characteristics are included and couplings of parameters are demonstrated explicitly in the multi-physics model. With changes in component structures and their integration forms, the trade-off of different machines can be achieved easily. Moreover, design codes are integrated into the model in this paper. Therefore, significant expertise for each component is not required for integrators to implement and shrink the component range to a reduced number of candidate technologies in system-level studies [24].

\subsection{MDO-Based Model Simplification}

To satisfy the system-level design demands, the MDO method is first used in the simplification of the multi-physics model to generate the simplified model, so that secondary-level models with characteristics of accuracy, simplicity, and consistency can be obtained. This process is illustrated in Figure 3.

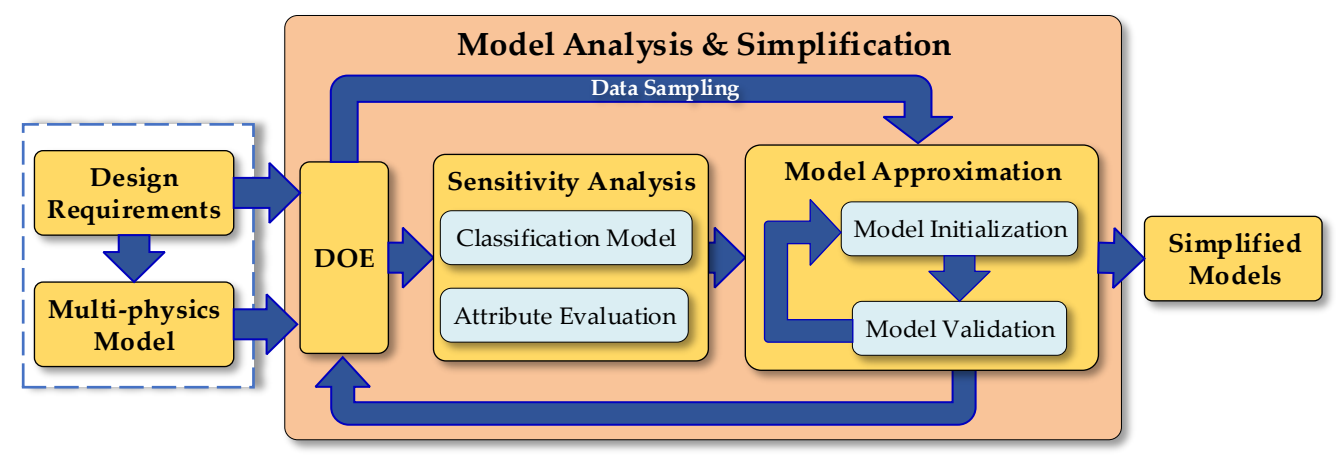

Figure 3. Multi-disciplinary design optimization (MDO)-based simplification of the multi-physics model.

By manipulating model inputs according to pre-specified design space, design of experiments (DOE) technology is applied to generate the data sample for the analysis. To describe the whole performance of the multi-physics model effectively, sufficient design points evenly spread in the design space are required. Then the model-based sensitivity analysis method is introduced to explore the design space and find cause-and-effect relationships between the inputs and outputs of the multi-physics model. From the sensitivity analysis, an optimal parameter combination for system-level machine models can be concluded. Based on the data and the optimal selected variable combination, surrogate models, which have been widely used in engineering design due to the low computational cost and high fidelity [36,37], are built for the system-level study. The approximation model can be improved by using better learners and adding additional sample points to achieve better precision.

\section{Modular Multi-Physics Model}

The variable-speed variable-frequency (VSVF) electrically excited synchronous generator is chosen as the case study to illustrate the improved multi-physics modeling process. The $2 \mathrm{D}$ structure of the machine is shown in Figure 4. In this study, the weight and power loss are naturally selected as the objectives. According to the proposed method and [29,35], the improved multi-physics model of machines includes the sub-models as discussed in the following. 


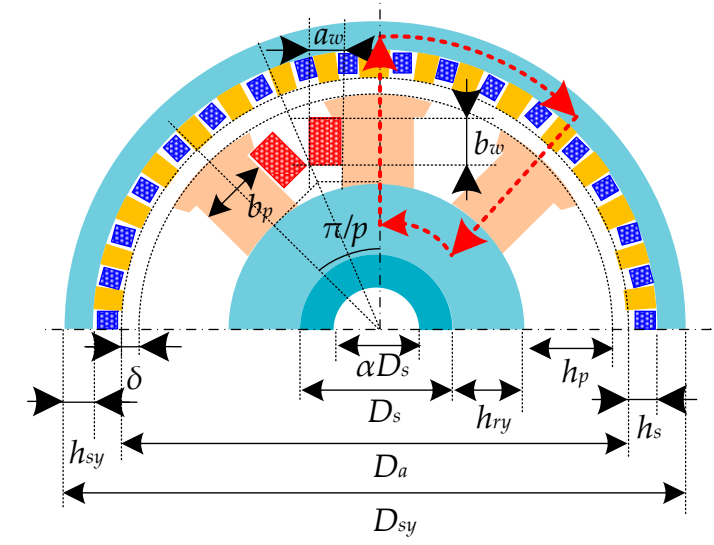

Excitation winding 䁂聞 Armature winding .....-) Magnetic circuit

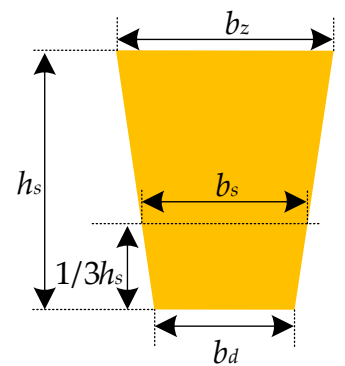

Tooth characteristics

Figure 4. Structure of an electrically excited synchronous generator.

\subsection{Shaft Model}

\subsubsection{Multi-Physics Coupled Constraints}

The shaft model involves mechanical, structural, and cooling features. The shaft is assumed to be a hollow cylinder, the diameter of which is determined by the electromagnetic power $P_{e m}$, rotational speed $n$, and the mechanical properties of the material:

$$
D_{s}=\max \left(\begin{array}{c}
A_{s h} \sqrt[3]{P_{e m} /\left[\left(1-\alpha_{s}^{4}\right) n\right]} \\
B_{s h} \sqrt[4]{P_{e m} /\left[\left(1-\alpha_{s}^{4}\right) n\right]} \\
\sqrt[3]{F /\left[0.1\left(1-\alpha_{s}^{4}\right)\left[\sigma_{-1}\right]_{b}\right]}
\end{array}\right) \times 10^{-3}
$$

where $D_{s}$ is the outer diameter; $A_{s h}$ is the coefficient of shear stress; $B_{s h}$ is the stiffness coefficient of material; $\left[\sigma_{-1}\right]_{b}$ is the admissible bending stress; $\alpha_{s}$ is the ratio of the inner to outer diameter, and $F$ is the equivalent moment.

$P_{\text {em }}$ can be calculated according to Equations (2) and (3) [38]:

$$
\begin{gathered}
P_{e m}=\left\{\begin{array}{l}
K_{E} P / \cos \varphi, \text { synchronous generator } \\
K_{E} P / \cos \varphi / \eta, \text { synchronous } / \text { induction motor }
\end{array}\right. \\
K_{E}=0.931+0.0108 \ln P-0.013 p,
\end{gathered}
$$

where $P$ is the rated power; $p$ represents the pole pairs; $\eta$ is the assumed efficiency; $\cos \varphi$ is the power factor, and $K_{E}$ is the per-unit value of full-load potential, namely, the ratio of induced electromotive force to the output voltage at full loads.

The gravity center of the rotor is assumed to be at the center of the shaft, and to be at the same position as the circle center of the stator. Thus, $F$ can be calculated according to Equation (4):

$$
\left\{\begin{array}{l}
F=\sqrt{F_{w}^{2}+0.59 F_{t}^{2}} \\
F_{w}=2.45 W_{r} l_{e f} \\
F_{t}=9.55 P_{c} / n
\end{array},\right.
$$

where $l_{e f}$ is the efficient length of machine; $F_{w}$ is the bending stress caused by the rotor weight $W_{r}$, and $F_{t}$ is the rated torque. 


\subsubsection{Design Goals}

The shaft weight $W_{s h}$ is shown in Equation (5):

$$
\left\{\begin{array}{l}
V_{s h}=\pi\left(1-\alpha_{s}^{2}\right) D_{s}^{2} l_{e f} / 4 \\
W_{s h}=\rho_{s h} V_{s h}
\end{array},\right.
$$

where $V_{s h}$ is the shaft volume, and $\rho_{s h}$ is the material density.

For the VSVF system, the influences of the variable rotational speed should be taken into consideration to ensure security, e.g., the stresses of shaft diameters.

\subsection{Yoke Model}

\subsubsection{Multi-Physics Coupled Constraints}

The yoke model involves magnetical, structural, and thermal features. As one of the main dimensions of a hollow cylinder, the yoke's thickness $h_{y}$ can be calculated according to the magnetic flux $\Phi_{y}$ and the selected induction $B_{y}$. The magnetic potential $F_{y}$ can be obtained according to the $B-H$ curve of the selected material:

$$
\left\{\begin{array}{l}
h_{y}=\Phi_{y} /\left(2 l_{e f} k_{c} B_{y}\right) \\
F_{y}=k_{c} H_{y}\left(2 h_{y}+\pi D_{y} / p\right) \xi
\end{array},\right.
$$

where $k_{c}$ is the lamination factor [39]; $D_{y}$ is the average diameter of the yoke; $\xi$ is the length coefficient of the yoke magnetic circuit, and $H_{y}$ is the yoke magnetic intensity corresponding to $B_{y}$.

It is assumed that $B_{y 0}$ is the yoke induction when the machine is in an idle run, whereas $B_{y N}$ is the yoke induction when the machine runs at full load. The relationship between $B_{y_{0}}$ and $B_{y N}$ is shown in Equation (7). By Equation (6) and (7), magnetic potentials of the yoke at no load and full load can be calculated. Combined with the magnetic potentials of other components calculated in a similar way, the magnetic circuit can be integrated, and the design requirements of the excitation windings can be obtained.

$$
B_{y N}=\left|E_{i}\right| B_{y 0},
$$

where $\left|E_{i}\right|$ is the amplitude of the per-unit value of rated induced electromotive force, and the calculation is introduced in the winding component.

The thermal circuit of the yoke satisfies Equation (8). Note that other components have similar thermal models.

$$
\left\{\begin{array}{l}
T_{y}=P_{\text {iron } \_y} R_{y} \\
\int^{2} P_{y} d t=C_{y} \Delta T_{y} \\
P_{y \_o}=P_{\text {iron } \_y}-P_{h_{-} y} \\
C_{y}=S p C_{y} m_{y}
\end{array},\right.
$$

where $T_{y}$ is the temperature rise of the yoke; $P_{\text {iron_ } y}$ is the produced heat by the yoke iron loss; $R_{y}$ is the thermal resistance of the yoke, which is dependent on the material and proportional to the surface area [20]; $P_{h-y}$ is the stored thermal power of the yoke; $C_{y}$ is the yoke thermal capacity, which is the product of the specific heat $S p C_{y}$ and mass $m_{y} ; \Delta T_{y}$ is the fluctuation of $T_{y}$, and $P_{y_{-} o}$ is the thermal power transferring from the yoke to other components.

At the steady heat balance state, as the yoke temperature is fixed, namely $\Delta T_{y}=0, P_{y_{-} o}$ equals $P_{\text {iron } \_}$, which indicates that the branch of $C_{y}$ is ignored in the thermal circuit in the design phase.

\subsubsection{Design goals}

The yoke weight $W_{y}$ and the iron loss $P_{y}$ can be calculated according to Equation (9): 


$$
\left\{\begin{array}{l}
W_{y}=\pi \rho_{y} l_{e f} D_{y} h_{y} / 2 \\
p_{y}=p_{10 / 50 \_y} B_{y}^{2}(f / 50)^{1.3} \\
P_{y}=k_{a \_} p_{y} W_{y}
\end{array},\right.
$$

where $\rho_{y}$ is the density of the yoke material; $p_{y}$ is the yoke iron loss per kilogram; $f$ is the operating frequency; $p_{10 / 50 \_y}$ is the yoke iron loss when $B=1 \mathrm{~T}$ and $f=50 \mathrm{~Hz}$, and $k_{a_{-} y}$ is the empirical coefficient, usually determined by Equation (10) [35]

$$
k_{a_{-} y}=\left\{\begin{array}{l}
3.6, \text { DC machine or } P<100 \mathrm{kVA} \text { AC machine } \\
1.3, P>100 \mathrm{kVA} \text { AC machine }
\end{array} .\right.
$$

\subsection{Slot Model}

\subsubsection{Multi-Physics Coupled Constraints}

The slot model involves structural, magnetical, and thermal features. The shape of slots is selected based on the rated power $P$ and armature winding voltage $E$ by experience; see Equation (11) [38]:

$$
\left\{\begin{array}{l}
E>1000 \mathrm{~V} \Rightarrow \text { open slot } \\
E<1000 \mathrm{~V} \& P>30 \mathrm{~kW} \Rightarrow \text { semi-open slot } \\
E<500 \mathrm{~V} \& P \leq 30 \mathrm{~kW} \Rightarrow \text { semi-closed slot }
\end{array}\right.
$$

where it is assumed that the slot induction $B_{S}$ equals the induction at a one-third height of the slot from the dedendum; see Figure 4. According to Equation (12), the magnetic potential of slots $F_{S}$ can be calculated:

$$
\left\{\begin{array}{l}
b_{s}=\pi D_{a} B_{\delta} /\left(2 Z k_{c} B_{s}\right) \\
F_{s}=2 k_{c} H_{s} h_{s}
\end{array},\right.
$$

where $Z$ is the slot number; $b_{s}$ and $H_{s}$ are, respectively, the tooth width and magnetic intensity corresponding to $B_{s}$, and $h_{s}$ is the slot height.

The slot inductions at no load and full load $B_{s 0}$ and $B_{s N}$ satisfy

$$
B_{S N}=\left|E_{i}\right| B_{S 0}
$$

\subsubsection{Design Goals}

Sections of every slot are assumed to be trapezoidal; thus, the slot weight $W_{s}$ and the iron loss $P_{S}$ can be calculated according to Equation (14):

$$
\left\{\begin{array}{l}
W_{s}=Z \rho_{y} l_{e f}\left(b_{Z}+b_{d}\right) h_{s} / 2 \\
Z=2 m p N_{Z} \\
b_{d} /\left(D_{a} / 2+h_{s}\right)=2 b_{Z} / D_{a}=b_{s} /\left(D_{a} / 2+2 h_{s} / 3\right) \\
p_{s}=p_{10 / 50 \_s} B_{s}^{2}(f / 50)^{1.3} \\
P_{s}=k_{a_{-} s} p_{s} W_{s}
\end{array}\right.
$$

where $\rho_{s}$ is density of the yoke material; $b_{z}$ and $b_{d}$ are the widths of the addendum and dedendum respectively; $N_{Z}$ is the slot number per pole per phase; $m$ is the phase number; $D_{a}$ is the bore diameter; $p_{s}$ is the slot iron loss per kilogram; $p_{10 / 50 \_s}$ is the yoke iron loss when $B=1 \mathrm{~T}$ and $f=50 \mathrm{~Hz}$, and $k_{a_{-} s}$ is the empirical coefficient, usually determined by Equation (15) [35]:

$$
k_{a_{\_} s}=\left\{\begin{array}{l}
4, \text { DC machine } \\
1.8, \text { induction machine } \\
2, P<100 \mathrm{kVA} \text { synchronous machine } \\
1.7, P>100 \mathrm{kVA} \text { synchronous machine }
\end{array},\right.
$$




\subsection{Air-Gap Model}

\section{Multi-Physics Coupled Constraints}

The air-gap model involves structural, magnetical, and thermal features. The air-gap thickness $\delta$, coefficient $k_{\delta}$, and magnetic potential $F_{\delta}$ are illustrated in Equation (16) [38]:

$$
\left\{\begin{array}{l}
\delta=0.36 A \tau /\left[\left(x_{d}^{*}-1\right) \Phi_{\delta}\right] \times 10^{-6} \\
k_{\delta}=\left(\pi D_{a} / Z+10 \delta\right) /\left(b_{Z}++10 \delta\right) \\
F_{\delta}=k_{\delta} B_{\delta} \delta / \mu_{0}
\end{array},\right.
$$

where $A$ is the electric loading; $\tau$ is the pole pitch; $x_{d}^{*}$ is the relative direct-axis inductance, ranging from 1.1 to $1.4 ; \Phi_{\delta}$ is the air-gap magnetic flux; $\mu_{0}$ is the permeability of vacuum, and $B_{\delta}$ is the air-gap induction:

$$
B_{p N}=\left|E_{i}\right| B_{p 0},
$$

where $B_{\delta 0}$ and $B_{\delta N}$ are the air-gap inductions at no load and full load.

As the oil spray cooling method is chosen for this machine, the equivalent thermal circuit of cooling oil is included in the thermal model of the air gap. To simplify the calculation, it is assumed that the oil is coated evenly on the surfaces of the stator and rotor.

\subsection{Pole Model}

\subsubsection{Multi-Physics Coupled Constraints}

The pole model involves magnetical, thermal, and structural features. The shape of the poles is determined by the machine type.

In this article, as the study case is a salient pole synchronous machine, the pole shape is illustrated in Figure 4. It is assumed that the pole shoes are of the same circle center as the rotor yoke, and its width is assumed to be equal to $\tau$. Then, the pole-core width $b_{p}$ can be calculated by exciting flux $\Phi_{p}$ and selected pole induction $B_{p}$. The magnetic potential of pole $F_{p}$ can be calculated by the corresponding magnetic intensity $H_{p}$ and pole height $h_{p}$. Therein, $B_{p}$ has the same meaning as the afore-mentioned $B_{y}$; see Equation (19):

$$
\begin{aligned}
& \left\{\begin{array}{l}
b_{p}=\Phi_{p} /\left(B_{p} l_{e f} k_{c}\right) \\
F_{p}=k_{c} H_{p} h_{p}
\end{array},\right. \\
& B_{p N}=\left|E_{i}\right| B_{p 0},
\end{aligned}
$$

where $B_{p 0}$ and $B_{p N}$ are the pole inductions at no load and full load, respectively.

For a PMSG, the volume of the permanent magnet $V_{m}$ can be estimated by the Larionoff method; see Equation (20) [38]. For the non-salient pole synchronous generator, the volume is calculated via the unsaturated vectogram, which is omitted here.

$$
V_{m}=\left.K_{b h} P\right|_{\cos \varphi=0} K_{\sigma 0} K_{a d F} K_{K}^{2} /\left(f B_{r} H_{C}\right) /\left(K_{K}-1\right),
$$

where $K_{b h}$ is a coefficient related to the material and selected operating point of the poles, and pole-arc coefficient, etc.; $\left.P\right|_{\cos \varphi=0}$ is the rated power when the power factor is zero; $K_{\sigma 0}$ is the leakage flux coefficient at no load, and is dependent on the rotor structure, material, and pole pairs; $K_{a d F}$ is the equivalent coefficient of armature potential, which is dependent on the pole material; $B_{r}$ and $H_{C}$ are the remanent induction and coercive force of the pole material, respectively; $K_{K}$ is the short-circuit current ratio.

Detailed dimensions can be calculated according to the pole structure (shape and magnetization direction); the process is omitted here but can be found in [35]. 
The selection of the operating point of poles is based on the material, including its magnetic and thermal characteristics. It is assumed that the demagnetization curve of the selected material is linear; thus, the operating point of poles $\left(B_{m}, H_{m}\right)$ can be calculated by Equations (21) and (22):

$$
\left\{\begin{array}{l}
B_{r T}=B_{r 0}\left[1-\left|\alpha_{B}\right|\left(T-T_{0}\right)\right] \\
H_{C T}=H_{C 0}\left[1-\left|\alpha_{B}\right|\left(T-T_{0}\right)\right] \\
\alpha_{B}=\left(B_{r h}-B_{r l}\right) / B_{r l} /\left(T_{h}-T_{l}\right)
\end{array},\right.
$$

where $B_{r T}$ and $H_{C T}$ are the remanent induction and coercive forces at temperature $T$, respectively; $B_{r 0}$ and $H_{C 0}$ are the remanent induction and coercive forces at standard temperature $T_{0}$, respectively; $\alpha_{B}$ is the temperature coefficient of remanence; $T$ is the actual operating temperature; $T_{h}$ and $T_{l}$ are the upper and lower limits of the allowed temperature, respectively, and $B_{r h}$ and $B_{r l}$ are the inductions at $T_{h}$ and $T_{l}$, respectively. The influence of temperature on the irreversible magnetic degradation is ignored here.

$$
\left\{\begin{array}{l}
B_{m}=K_{\sigma} B_{r T} / 2 \\
H_{m}=\left(1-K_{\sigma} / 2\right) H_{C T}
\end{array},\right.
$$

where $K_{\sigma}$ is the leakage flux coefficient.

\subsubsection{Design Goals}

The pole weight $W_{p}$ and the iron loss $P_{p}$ can be calculated according to Equation (23):

$$
\left\{\begin{array}{l}
W_{p}=\rho_{p} V_{m} \\
p_{p}=p_{10 / 50 \_p} B_{p}^{2}(f / 50)^{1.3} \\
P_{p}=p_{p} W_{p}
\end{array},\right.
$$

where $\rho_{p}$ is the density of the pole material; $p_{p}$ is the pole iron loss per kilogram, and $p_{10 / 50 \_p}$ is the pole iron loss when $B=1 \mathrm{~T}$ and $f=50 \mathrm{~Hz}$.

\subsection{Winding Model}

\subsubsection{Multi-Physics Coupled Constraints}

The winding model involves electromagnetic and thermal features. The configurations of the armature winding are determined by optimally selecting the magnet wire based on the carrying capacity, which is calculated by the rated power $P$, the armature winding voltage $E$, the pitch ratio $\beta$, and the current density $J_{a}$, whereas those of the excitation winding is determined by the excitation magnetic potential $F_{f}$ and current density $J_{f}$.

In detail, the number of winding turns in series $N$ is determined by $E$, and the number of paralleled conductors $a$ can be calculated according to the standard MIL-W-5088L and the wire gauge standard [40]; see Equation (24):

$$
\left\{\begin{array}{l}
E=4.44 f N k_{W} \Phi \\
k_{W}=\sin (\pi \beta / 2) \sin (q \alpha / 2) \\
q=Z /(2 p m), \alpha=2 \pi p / Z \\
I=\pi a J d^{2} k_{s h} k_{g} k_{T}
\end{array}\right.
$$

where $f$ is the frequency of the output voltage; $k_{W}$ is the winding coefficient; $\Phi$ is the main magnetic flux; $\beta$ is the ratio of the winding pitch to the polar pitch $\tau ; q$ is the number of slots per pole per phase; $\alpha$ is the electrical angle; $m$ is the phase number; $J$ is the current density of the selected wire gauge; $d$ is the diameter of the selected wire gauge; $k_{s h}$ is the bundling coefficient of conductors; $k_{g}$ is the altitude correction coefficient of conductors, and $k_{T}$ is the temperature coefficient of conductors. 
According to the requirements of the wire insulation [41], the diameter of the selected electromagnetic wire $d_{e w}$ satisfies Equation (25):

$$
\left\{\begin{array}{l}
t h_{i n s}=E / \varepsilon_{e w} \\
d_{e w}=d+2 t h_{i n s} \\
d_{e w} \leq d_{\max }
\end{array},\right.
$$

where $d_{\max }$ is set as the maximum wire diameter allowed for an inlay technology reason, and $\varepsilon_{e w}$ is the dielectric strength of the insulation.

According to the first-order model of the machine [42], the resistance and inductance can be calculated; see Equations (26) and (27):

$$
\left\{\begin{array}{l}
R_{a}=k_{r} N \rho_{c} l_{c} / s_{c} / a \\
\rho_{c}=\rho_{0}\left(1+\alpha_{c} \Delta T\right)
\end{array}\right.
$$

where $R_{a}$ is the resistance of the armature winding; $k_{r}$ is the coefficient considering the skin effect, which is dependent on the shape, configuration, and frequency of conductor; $\rho_{c}$ is the electrical resistivity of the conductor, which is dependent on the material (electrical resistivity at $20^{\circ} \mathrm{C} \rho_{0}$ and temperature coefficient $\alpha_{c}$ ) and temperature rise $\Delta T$, and $l_{c}$ and $s_{c}$ are the average conductor length and area per turn, respectively.

$$
\left\{\begin{array}{l}
X_{m}=4 f \mu_{0} N^{2} l_{e f} \lambda_{m} /(p q) \\
X_{M}=-4 f \mu_{0} N^{2} l_{e f} \lambda_{M} /(p q) \\
X_{\sigma}=4 f \mu_{0} N^{2} l_{e f}\left(\lambda_{s}+\lambda_{\delta}+\lambda_{t}+\lambda_{E}\right) /(p q) \\
X_{a}=X_{m}-X_{M}+X_{\sigma}
\end{array},\right.
$$

where $X_{m}, X_{M}, X_{\sigma}$, and $X_{a}$ are the main, mutual, leakage, and total inductances of the armature winding, respectively; the specific permeance coefficients $\lambda_{m}, \lambda_{M}, \lambda_{s}, \lambda_{\delta}, \lambda_{t}$, and $\lambda_{E}$ are dependent on the slot shape and configurations of windings; detailed expressions are omitted here due to the existence of multiple kinds of combined constructs [35].

To integrate the magnetic circuit, the per-unit value of the rated induced electromotive force $E_{i}$, which is a gain coefficient to obtain the rated operating points of every component, is calculated according to the impedance of armature windings [29]; see Equation (28):

$$
\left\{\begin{array}{l}
R_{a}^{*}=R_{a} P / E^{2}, X_{\sigma}^{*}=X_{\sigma} P / E^{2} \\
E_{i}=W+\mathrm{j} Q=\sqrt{W^{2}+Q^{2}} e^{j \varepsilon} \\
W=1+R_{a}^{*} \cos \varphi+X_{\sigma}^{*} \sin \varphi \\
Q=X_{\sigma}^{*} \cos \varphi-R_{a}^{*} \sin \varphi \\
\varepsilon=\arctan (Q / W)
\end{array}\right.
$$

where $R_{a}^{*}$ and $X_{\sigma}^{*}$ are the per-unit value of $R_{a}$ and $X_{\sigma}$, respectively; $W$ and $Q$ are the real and imaginary parts of $E_{i}$, respectively, and $\varepsilon$ is the phase angle of $E_{i}$.

In addition, the winding thermal model includes the thermal circuit of both wire and insulation.

\subsubsection{Design Goals}

According to the determined strands of wires, the weight $W_{a w}$ and power $\operatorname{loss} P_{a w}$ can be calculated; see Equation (29):

$$
\left\{\begin{array}{l}
W_{a w}=m N a W_{a w \_s} \\
W_{a w \_s}=\pi\left(l_{e f}+\tau\right)\left[\rho_{e w} d^{2}+\rho_{i n s}\left(d_{e w}^{2}-d^{2}\right)\right] / 2 \\
P_{e w}=m I^{2} R_{a}
\end{array}\right.
$$


where $W_{a w \_s}$ is the weight of single-strand wire, and $\rho_{e w}$ and $\rho_{i n s}$ are the densities of conductor and insulation materials, respectively.

\subsection{Integration Model}

\subsubsection{Components Coupled Constraints}

The whole machine model is integrated into aspects of structural, magnetical, and thermal features.

The structural integration is mainly focused on the radial dimensions of components, including the main geometrical parameter shown in Equation (30), constraints of lengths shown in Equation (31), and the space-filling of windings shown in Equation (32):

$$
\left\{\begin{array}{l}
C A=D_{a}^{2} l_{e f} /\left(P_{e m} / n\right)=6.1 /\left(\alpha^{\prime} k_{W} A B_{\delta}\right) \\
\lambda=l_{e f} / \tau \\
D_{s y}=D_{a}+K_{p} \tau
\end{array},\right.
$$

where $C A$ is the machine constant; $D_{a}$ is the diameter of the armature; $\lambda$ is the dimension ratio; the pole-arc coefficient $\alpha^{\prime}$ is assumed to be $2 / \pi$, which results in a sinusoidal magnetic field; $D_{s y}$ is the stator outer diameter, and $K_{p}$ is an empirical coefficient related to $p$.

As component dimensions are calculated by their respective inductions, when components are integrated into the structure, implicit constraints exist among inductions of components to make sure that they can be put in a limited space. Therefore, component inductions should be properly selected to satisfy Equations (31) and (32):

$$
\begin{gathered}
\left\{\begin{array}{l}
h_{s}=\left(D_{s y}-D_{a}\right) / 2-h_{s y}>0 \\
h_{p}=\left(D_{a}-D_{s}\right) / 2-h_{r y}>0
\end{array},\right. \\
\left\{\begin{array}{l}
b_{p} / 2+a_{w}+b_{w} \tan \theta=r_{r} \sin \theta \\
0<b_{w} \leq r_{r} \cos \theta-\left(D_{s} / 2+h_{r y}\right) \\
r_{r}=D_{s} / 2+h_{r y}+h_{p} \\
\theta=\pi / 2 / p \\
40 \% \leq K \leq 80 \%
\end{array}\right.
\end{gathered}
$$

where $h_{s y}$ and $h_{r y}$ are the thicknesses of the stator and rotor yoke, respectively; $a_{w}$ and $b_{w}$ are the tangential and radial lengths of excitation windings, respectively; $r_{r}$ is the radius of the rotor; $\theta$ is one-quarter of the mechanical angle of machine, and $K$ is the slot fill factor.

The magnetical integration calculates the magnetic circuit, and the excitation requirement is obtained by adding the no-load and full-load magnetic potentials of components; see Equation (33):

$$
\left\{\begin{array}{l}
F_{a}=1.1\left(F_{\delta}+F_{s}+F_{r y}+F_{p}+F_{s y}\right) \\
F_{f}=1.05 F_{a} \sqrt{1+\left(F_{a d} / F_{a}\right)^{2}+2 F_{a d} / F_{a} \sin (\varphi+\varepsilon)}
\end{array},\right.
$$

where $F_{r y}$ and $F_{s y}$ are the magnetic potentials of rotor yoke and stator yoke, respectively; $F_{a}$ is the magnetic motive force of armature winding, and $F_{a d}$ is the direct-axis magnetic motive force of armature winding; detailed expressions are omitted here as this is a complex equation.

The thermal integration illustrates the thermal behavior of every component (slot, yoke, etc.) with respect to their resistances, capacities, and ambient conditions, by a thermal circuit, which is coupled with electromagnetic characteristics through losses; see Figure 5. Requirements of the cooling system, e.g., the flow rate of the cooling oil [43], can be deduced from Equation (6) by calculating the thermal resistance and capacity of cooling oil $R_{c o}$ and $C_{c o}$, which offers a resting interface to the fuel pump system. In addition, the thermal load $A J_{a}$ is also given as a constraint here. 


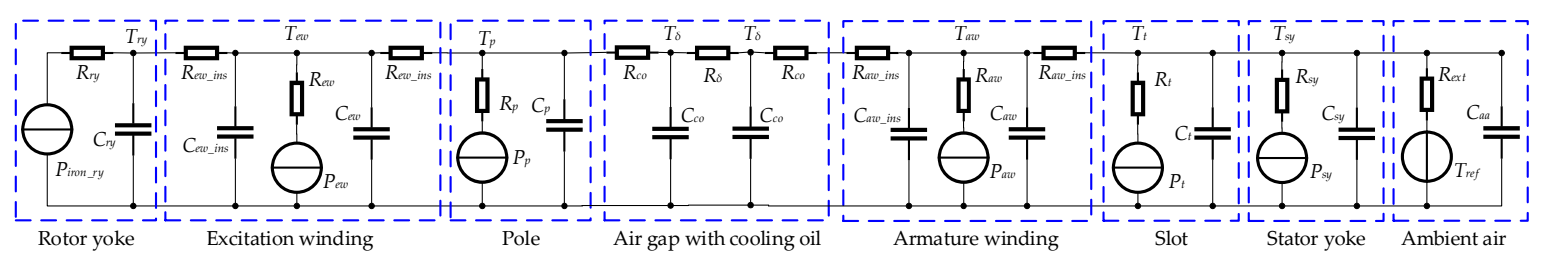

Figure 5. The simplified lumped thermal model of the electrically excited synchronous generator.

\subsubsection{Design Goals}

The total weight $W_{G}$ and power loss $P_{G}$ are calculated by Equation (34):

$$
\left\{\begin{array}{l}
W_{G}=W_{s h}+W_{r y}+W_{e w}+W_{p}+W_{s}+W_{a w}+W_{s y} \\
P_{G}=P_{f}+P_{a w}+P_{e w}+P_{r y}+P_{p}+P_{s}+P_{s y}+P_{\Delta}
\end{array},\right.
$$

where $W_{r y}, W_{e w}, W_{a w}$, and $W_{s y}$ are the weights of the rotor yoke, excitation windings, armature windings, and stator yoke, respectively; $P_{e w}$ and $P_{a w}$ are the copper loss of excitation and armature windings, respectively; $P_{r y}$ and $P_{s y}$ are the iron loss of the rotor and stator yoke, respectively; $P_{\Delta}$ is the stray loss, and is assumed to be one percent of the rated power, and $P_{f}$ is the total friction loss; see Equation (35) [44].

Therein, the rotor is assumed to be a cylinder when the windage friction loss is calculated:

$$
\left\{\begin{array}{l}
P_{f}=P_{s b}+P_{a i r} \\
P_{s b}=1.05 F n \times 10^{-4} \\
P_{\text {air }}=\rho_{\text {air }}\left(D_{a}-2 \delta\right)^{4} \omega^{3}\left[K_{f 1} \pi l_{e f}+K_{f 2}\left(D_{a}-2 \delta\right) / 8\right] \\
\rho_{\text {air }}=1.293 p_{\text {ress }} /(1+0.00367 T) / 760
\end{array},\right.
$$

where $P_{s b}$ and $P_{a i r}$ are the friction losses of bearing and windage, respectively; $\rho_{a i r}$ is the air density at temperature $T\left({ }^{\circ} \mathrm{C}\right)$ and pressure $p_{\text {ress }}(\mathrm{mmHg}) ; \omega$ is the angular velocity of the rotor, and $K_{f 1}$ and $K_{f 2}$ are the friction coefficients of the cylinder and disk, and are dependent on the Couette Reynolds Number Re.

It is assumed that $K_{f 1}$ and $K_{f 2}$ are equal to $K_{f}$, and $K_{f}$ can be calculated by Equation (36) [45]:

$$
K_{f}=\left\{\begin{array}{l}
0.515 C_{r}\left[\delta /\left(D_{a}-2 \delta\right)\right]^{0.3} R e^{-0.5}, 500<R e<10^{4} \\
0.0325 C_{r}\left[\delta /\left(D_{a}-2 \delta\right)\right]^{0.3} R e^{-0.2}, \operatorname{Re}>10^{4}
\end{array}\right.
$$

where $C_{r}$ is the relative roughness coefficient of the rotor, and is assumed to be 1 , and $R e$ is obtained according to Equation (37):

$$
\operatorname{Re}=\left(D_{a}-2 \delta\right)^{2} \omega / v,
$$

where $v$ is the kinematic viscosity of the airflow.

\subsection{Summary}

\subsubsection{Problem Definition}

The machine design problem can be sorted into the forms illustrated in Equation (38) by the equations above.

$$
\begin{aligned}
& \operatorname{Min}\left[W_{G}, P_{G}\right] \\
& \text { s.t. } \\
& \text { Explicit : multi-physics couplings of components } \\
& \text { Implicit : }\left\{\begin{array}{l}
\text { structural couplings among components } \\
\text { initial assumptions verification }
\end{array}\right.
\end{aligned}
$$

where the weight, power loss, physical dimensions, and cost of the generator are usually the major design goals of a machine in aircraft applications. In this study, the weight and power loss are naturally 
selected as the objectives, and the physical dimension of the machine is given in the process of modeling. As the cost can be calculated by multiplying the material price by the weight [44], it is omitted here. Detailed calculations are included in the design goal of every modeling module.

As illustrated in the constraints of every modeling module, the machine has electromagnetic, structural, and thermal features, and the constraints of the problem include multi-physics couplings inside and among components. They can be divided into two kinds, namely, explicit and implicit. The explicit constraints are those expressions in which the outputs can be represented by inputs without iterative calculations, e.g., Equation (1), whereas implicit constraints are those expressions that cannot, e.g., Equation (31). Explicit constraints can be substituted into the goal functions directly, whereas implicit ones cannot. Therefore, considering that the search algorithm has the ability to achieve optimal solutions, if the implicit constraints are not satisfied, the model outputs high penalty factors to break the re-design loop to simplify the calculation.

\subsubsection{Model Improvements}

Compared with other models, the developed multi-physics model is improved as follows:

- The model is restructured and divided into several replaceable modules to illustrate the couplings of components. Thus, other machine types can be evaluated easily just by changes in shape, material, and radial positions of component modules, which also follows the concurrent design concept.

- Design codes, e.g., the wire gauge, shape selection experiences, and empirical formulas are embedded to realize easier designs for system integrators.

- Inputs are well selected according to the insulation requirements, which offer comprehensive links with practical design requirements, whereas penalty coefficients are used to break the closed loops of the design, which simplifies the calculation.

However, the dimensionality of the built model is greater than that of the model in [29]. This is because some design variables are not independent, e.g., component inductions. For the purpose of power system integration, this high-dimensional design space clearly leads to difficulties in the trade-off. Therefore, simplification is needed to improve the design efficiency.

\section{Model Simplification}

To further reduce the dimensionality of the built multi-physics model, this section explores its design space by sensitivity analysis methods and then establishes the simplified models used for system integration based on the model approximation technologies. To illustrate this process clearly, materials and empirical coefficients are pre-specified (the 45 carbon steel made shaft, the D41 cold-rolled silicon steel sheet, the fluoropolymer coating insulation, the ratio of the inner and outer diameter, and relative direct-axis inductance, etc.). Variation ranges of other undetermined design variables are listed in Table 1, according to the requirements of Class F insulation.

To implement the simplification, a data set, which can efficiently describe the whole performance of the multi-physics model, is required. To obtain the data set, the optimal Latin hypercube design (LHD) algorithm [46-48] is applied and the discrepancy of point sets is selected as the optimized criterion objective to generate a design matrix evenly spread in the design space presented in Table 1. To facilitate an accurate analysis, a matrix of 10,000 points is used here. The process is demonstrated in Figure 6. With the increase of design points, the discrepancy of point sets declines evidently first and then remains steady after 800 iterations. Then, by substituting the design points into Equation (38), corresponding outputs of the multi-physics model are obtained. As constraints are not always satisfied, 990 unsuitable points are eliminated before the analysis. As every iteration generates nine points on average, the remaining 9010 design points can be regarded as the results at the 1001st iteration in Figure 6, which verifies the distribution performance of this filtered data set. 
Table 1. Variation ranges of the built generator model.

\begin{tabular}{ccc}
\hline Design Variables & Lower Levels & Upper Levels \\
\hline$P(\mathrm{~kW})$ & 30 & 300 \\
$p$ & 1 & 6 \\
$\beta$ & $2 / 3$ & $7 / 8$ \\
$\cos \varphi$ & 0.8 & 0.9 \\
$n(\mathrm{r} / \mathrm{min})$ & 1000 & 30,000 \\
$\lambda$ & 0.6 & 4 \\
$\Delta T\left({ }^{\circ} \mathrm{C}\right)$ & 0 & 105 \\
$A(\mathrm{~A} / \mathrm{m})$ & $2.3 \times 10^{4}$ & $6 \times 10^{4}$ \\
$B_{p}(\mathrm{~T})$ & 1.3 & 1.7 \\
$B_{r y}(\mathrm{~T})$ & 1.15 & 1.7 \\
$B_{s t}(\mathrm{~T})$ & 1.5 & 2 \\
$B_{s y}(\mathrm{~T})$ & 1.35 & 1.75 \\
$B_{\delta}(\mathrm{T})$ & 0.6 & 1 \\
$J_{a}\left(\mathrm{~A} / \mathrm{mm}^{2}\right)$ & 7 & 11 \\
$J_{f}\left(\mathrm{~A} / \mathrm{mm}^{2}\right)$ & 4.5 & 9 \\
$E(\mathrm{~V})$ & 115 & 5000 \\
$A J_{a}\left(\mathrm{~A} / \mathrm{m} \cdot \mathrm{A} / \mathrm{mm}^{2}\right)$ & $2 \times 10^{5}$ & $5 \times 10^{5}$ \\
\hline & &
\end{tabular}

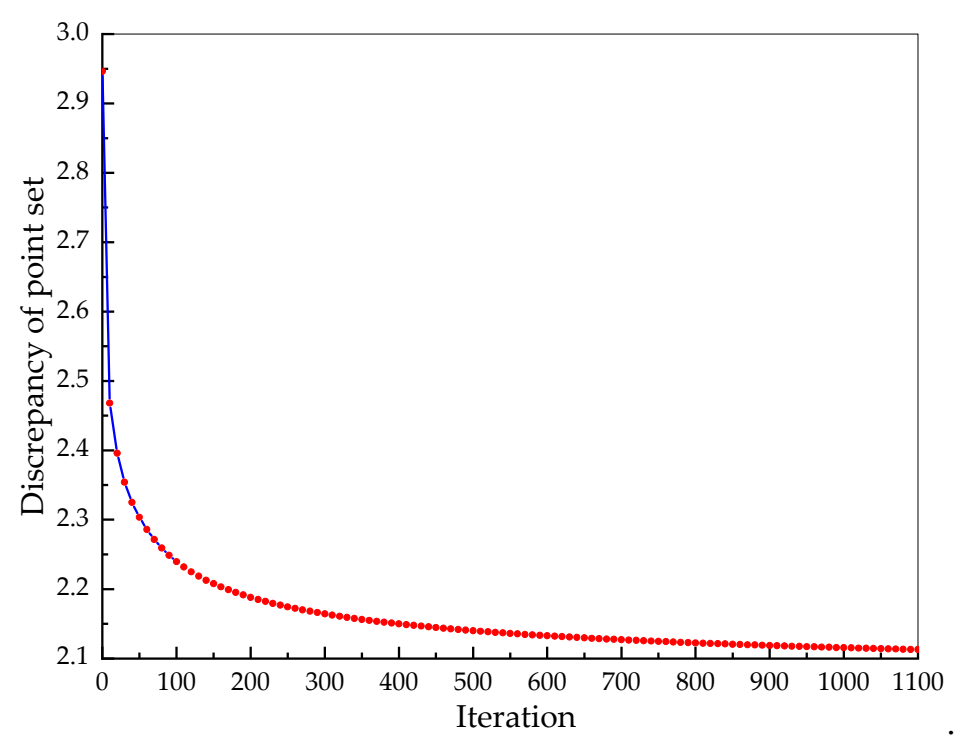

Figure 6. Optimal Latin hypercube design (LHD)-based design of experiments (DOE) process.

\subsection{Sensitivity Analysis}

To obtain the optimal parameter combination, this paper analyzes trends between inputs and outputs to identify key factors of the multi-physics model by the multiple quadratic regression method.

Equation (39) illustrates the principle of this method with a binary quadratic function. The differential of $y$ can be divided into main and interaction effects of $x_{1}$ and $x_{2}$. For example, coefficients $c_{1}$ and $2 c_{3}$ can reflect the main effects of $x_{1}$ on $y ; c_{2}$ and $2 c_{4}$ can reflect the main effects of $x_{2}$ on $y$, and $c_{5}$ can reflect the interaction effect of $x_{1}$ and $x_{2}$ on $y$. Therefore, based on the multiple quadratic regression model, the influences of design variables on the weight and power loss can be evaluated:

$$
\left\{\begin{array}{l}
y=c_{0}+c_{1} x_{1}+c_{2} x_{2}+c_{3} x_{1}^{2}+c_{4} x_{2}^{2}+c_{5} x_{1} x_{2} \\
d y=c_{1} d x_{1}+c_{2} d x_{2}+2 c_{3} d x_{1}+2 c_{4} d x_{2}+c_{5} d\left(x_{1} x_{2}\right)
\end{array}\right.
$$

The determination coefficient $\left(R^{2}\right)$ is used to evaluate the imitative effect of the multiple quadratic regression model. The $R^{2}$ of the weight and power loss models are 0.9705 and 0.97848 , respectively, which indicates the accuracy of the analysis. 
Figure 7 illustrates the main factor plots of weight and power loss. The main factors of the weight $W_{G}$ are the rotational speed $n$, rated power $P$, pole pairs $p$, and electric loading $A$, whereas those of the power loss $P_{G}$ are the rated power $P$, electric loading $A$, pole pairs $p$, rotational speed $n$, and air-gap induction $B_{\delta}$. Both weight and power loss rise linearly with the increase of the rated power. Moreover, with the rise of pole pairs, the weight declines whereas the power loss increases. In addition, it is notable that the voltage has few effects on weight and power loss.

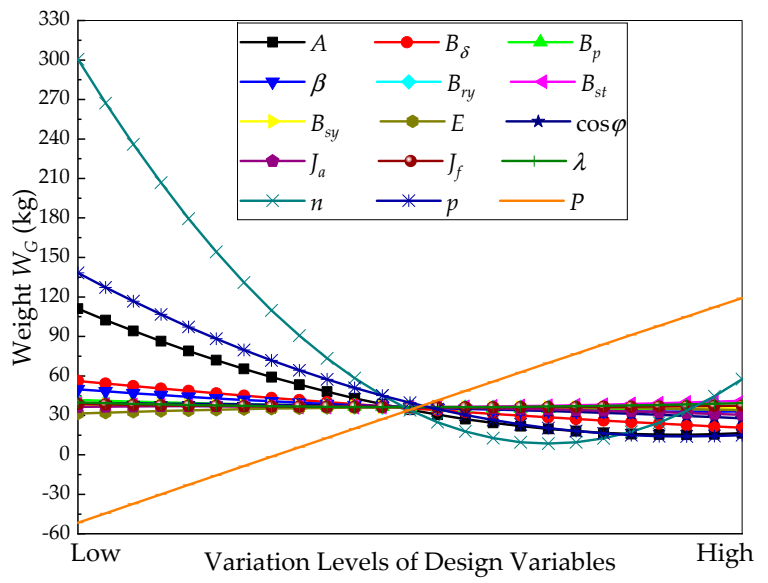

(a)

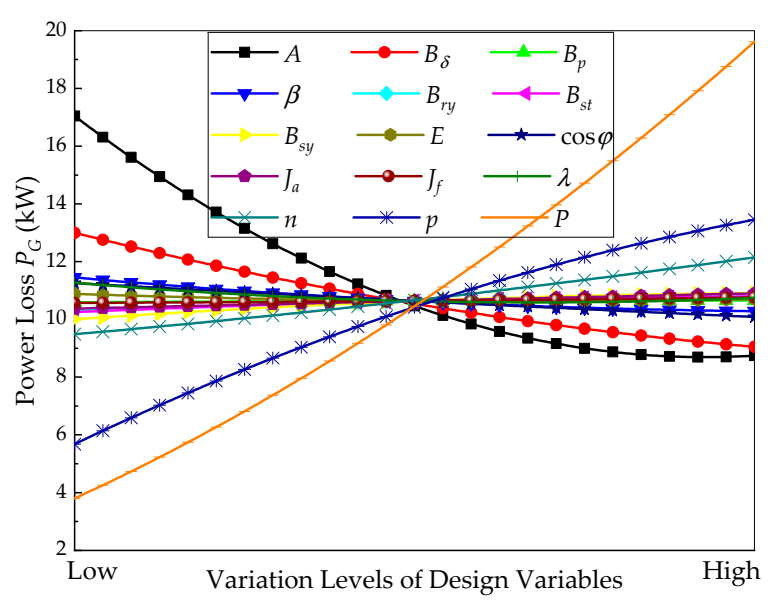

(b)

Figure 7. Main effect plots of the generator: (a) main effect plots for the weight; (b) main effect plots for the power loss.

Due to different dimensions and ranges of variables, dimensionless processing is executed to achieve fair identification of couplings; the top 20 contributors of design variables and couplings are shown in Figure 8. The weight attributes $83.05 \%$ of itself to the couplings of the rated power $P$, pole pairs $p$, rotational speed $n$, electric loading $A$, air-gap flux density $B_{\delta}$, and pitch ratio $\beta$, whereas the power loss attributes $79.35 \%$ of itself to the couplings of the rated power $P$, pole pairs $p$, rotational speed $n$, electric loading $A$, air-gap flux density $B_{\delta}$, pitch ratio $\beta$, and power factor $\cos \varphi$.

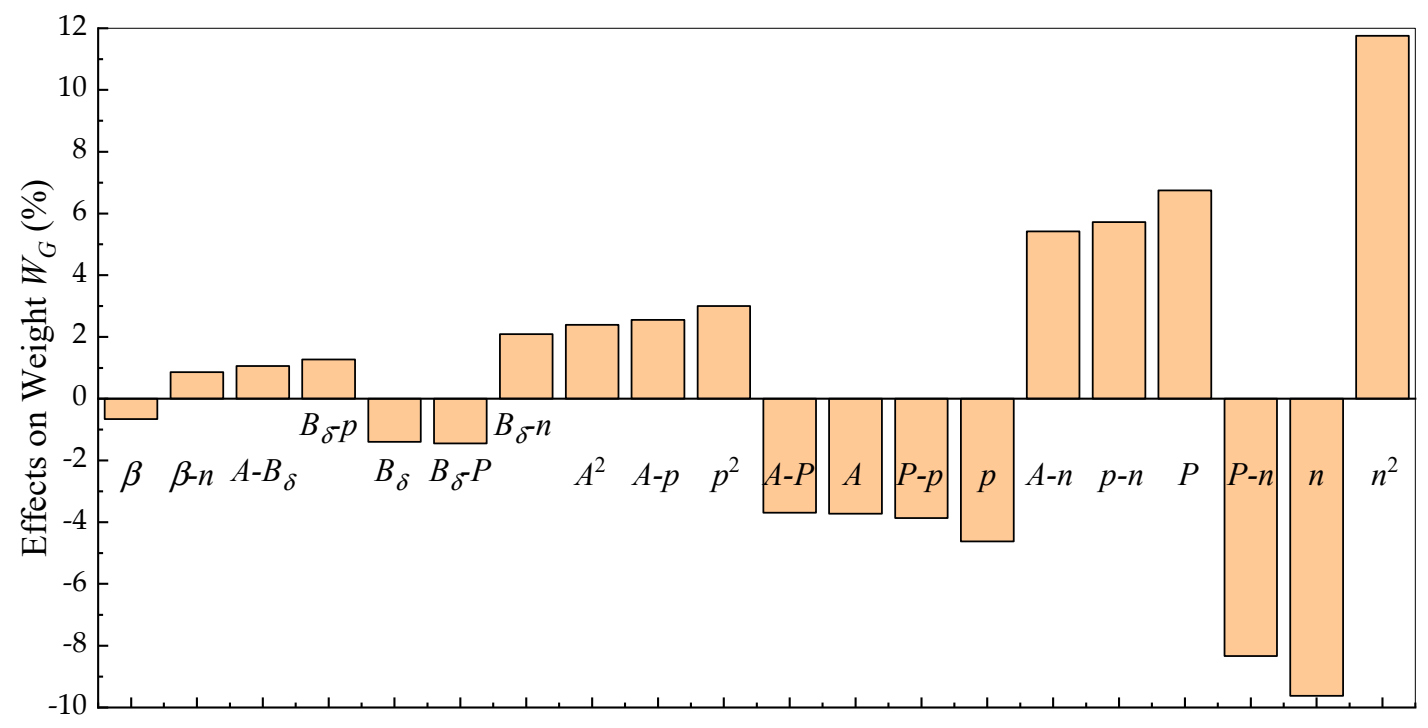

(a)

Figure 8. Cont. 


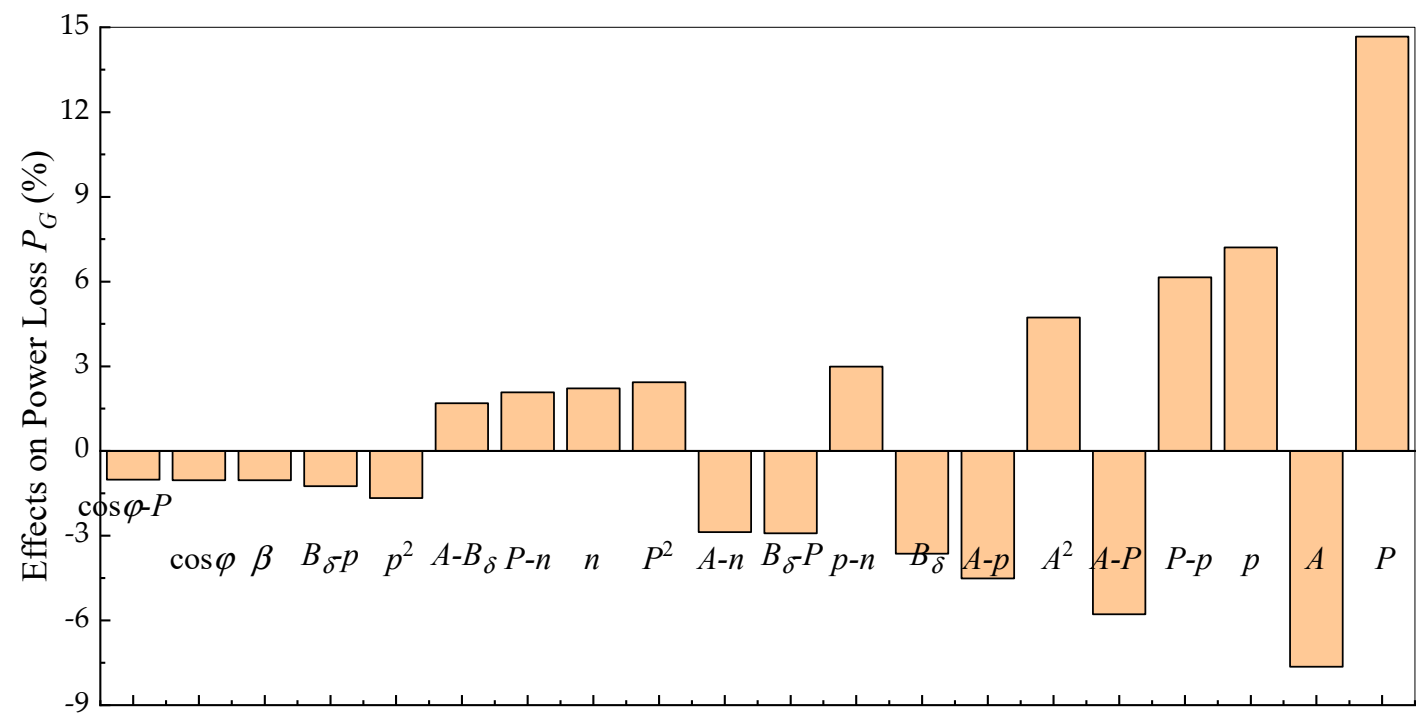

(b)

Figure 8. The top 20 contribution plots: (a) contributions to the weight; (b) contributions to the power loss. $x-y$ means the coupling between variable $x$ and variable $y$.

According to the analysis above, some design variables can be ignored, and model inputs before and after the dimensionality reduction are demonstrated in Table 2 . The dimensionalities of the weight and power loss models can be reduced from 15 to 6 and 7, respectively. As the input variables of the simplified model are the subset of those of the multi-physics model, they have a consistent interface. For a more comprehensive simplification, the pre-specified parameters can be added into the analysis.

Table 2. Candidate variables for simplified models.

\begin{tabular}{ccc}
\hline \multicolumn{2}{c}{ Models } & Alternative Inputs \\
\hline \multirow{2}{*}{ Before } & $W_{G}$ & $P, p, A, n, B_{\delta}, \beta, \cos \varphi, B_{p}, B_{r y}, B_{s t}$, \\
& $P_{G}$ & $B_{s y}, J_{a}, J_{f}, \lambda$, and $E$ \\
\multirow{3}{*}{ After } & $W_{G}$ & $P, p, A, n, B_{\delta}$, and $\beta$ \\
& $P_{G}$ & $P, p, A, B_{\delta}, n, \beta$, and $\cos \varphi$ \\
\hline
\end{tabular}

\subsection{Model Approximation}

According to the reduced input variables in Table 2, the methods of radial basis function (RBF) and quartic response surface model (RSM) are used for the approximations of the data sample generated from DOE. Ten-fold cross-validations are executed by randomly partitioning the sample into nine training subsets to train the model and one test subset to evaluate it.

Comparisons between the built models and the test subsets are illustrated in Table 3. The RSM-based weight model has a relatively low accuracy and high degree of uncertainty, whereas other models achieve satisfactory results. As only a small amount of data is required by the approximation of the reduced-order quartic RSM-based model, the sample containing 9010 points cannot be covered. Accordingly, although ten-fold cross-validation helps keep models accurate by calculating multiple surrogate models of different subsets, the performance of reduced-order RSM-based models is worthless for large sample data. In other words, RBF-based surrogate models are preferred. 
Table 3. Cross-validation error results of surrogate models.

\begin{tabular}{cccc}
\hline Models & $\begin{array}{c}\text { Evaluation } \\
\text { Indexes }\end{array}$ & RBF & RSM \\
\hline \multirow{2}{*}{$W_{G}$} & $R^{2}$ & 0.959 & 0.783 \\
& Average Error $^{1} / \%$ & 2.75 & 13.12 \\
& Max Error $^{1 / \%}$ & 18.29 & 29.29 \\
& RMSE $^{2}$ & 0.06 & 0.16 \\
$P_{G}$ & $R^{2}$ & 0.939 & 0.98 \\
& Average Error/\% & 6.701 & 2.44 \\
& Max Error/\% & 14.79 & 11.32 \\
& RMSE & 0.076 & 0.0394 \\
\hline
\end{tabular}

${ }^{1}$ Max and average error are both calculated by absolute values of relative errors. ${ }^{2}$. Root mean square errors (RMSE) are calculated after normalizations, varying from 0 to 1.

\section{Analysis}

\subsection{Verification}

To verify the modeling method, the auxiliary power unit generator (APUG) in a Boeing 787 with known parameters (apparent power $P_{s}=225 \mathrm{kVA}$, electric loading $A=60,000 \mathrm{~A} / \mathrm{m}$, pole pairs $p=2$, rotational speed $n$ varies in the range of 10,800-24,000 r/min, nominal speed $n_{N}=12,000 \mathrm{r} / \mathrm{min}$, air-gap induction $B_{\delta+}=0.8 \mathrm{~T}$, pitch ratio $\beta=0.75$, rated voltage $E=230 \mathrm{~V}$, weight $W_{G}=52 \mathrm{~kg}$, power loss $P_{G}=20 \mathrm{~kW}$ when $n=24,000 \mathrm{r} / \mathrm{min}$ and power factor $\cos \varphi=0.85$ ) [49] is used as the case study.

According to the method, the multi-level models are established. As some inputs of the multi-physics model, e.g., operating points $B_{p}, B_{r y}, B_{s t}, B_{s y}, J_{a}$, and $J_{f}$, and the dimension ratio $\lambda$, are not given, the uncertainties clearly result in a Pareto set. Therefore, according to ranges of the uncertain variables in Table 1, NSGA-II (non-dominated sorting genetic algorithm II) with 100 populations and 1000 generations is used, and the Pareto plot comprised of 3171 points obtained is illustrated in Figure 8. The design results of simplified models and the actual solution are also shown in Figure 9. Therein, two optimal solutions (M1 and M2) on the Pareto plot of the multi-physics model are given as examples for the convenience of the analysis.

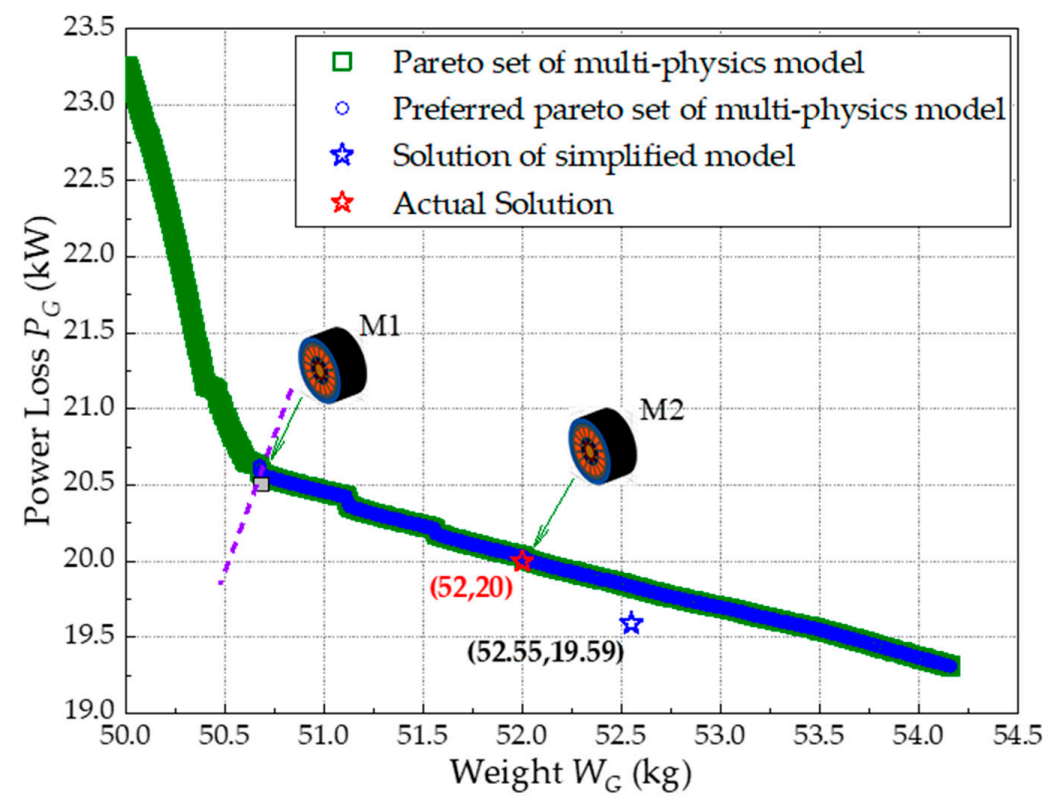

Figure 9. Pareto results of models and actual values. 


\subsubsection{Multi-Physics Model Analysis}

The actual solution is nearly located on the Pareto plot of the multi-physics model with the minimum errors $(0.006 \%$ of the weight and $2.7 \%$ of the power loss, compared with solution M2), which verifies the multi-physics model. The uncertainties lead the calculated weight to a variation ranging from $-3.82 \%$ to $4.15 \%$ and the power loss to a variation ranging from $-2.56 \%$ to $16.27 \%$ of the actual solution, which suggests that the weight of optimal solutions varies less than the power loss. Therefore, although the decision-making model is not studied here, more importance will be attached to the power loss by designers.

\subsubsection{Dimensionality Reduction Analysis}

To verify the dimensionality reduction of simplified models, data distributions of design variables of the optimal solutions are analyzed. As the power of the excitation system is relatively low and leads to few fluctuations of the results, their design variables are ignored here.

It is noteworthy that the Pareto set can be divided into two parts with the demarcation point M1 by the dimension ratio $\lambda$; see Figure 9 . Figure 10 demonstrates the data distribution of the dimension ratio $\lambda$ in the two parts. It can be concluded that a value of the dimension ratio $\lambda$ exists in the range from 1.263 to 1.267 as the extreme point of the weight model, whereas it exists in the range from 1.591 to 1.593 as the extreme point of the power loss model. The selection of the dimension ratio is determined by the designer, depending on which index is more important. According to the power loss-preferred design, solutions of the 1728 blue-colored circle points in Figure 9 are probably better choices.

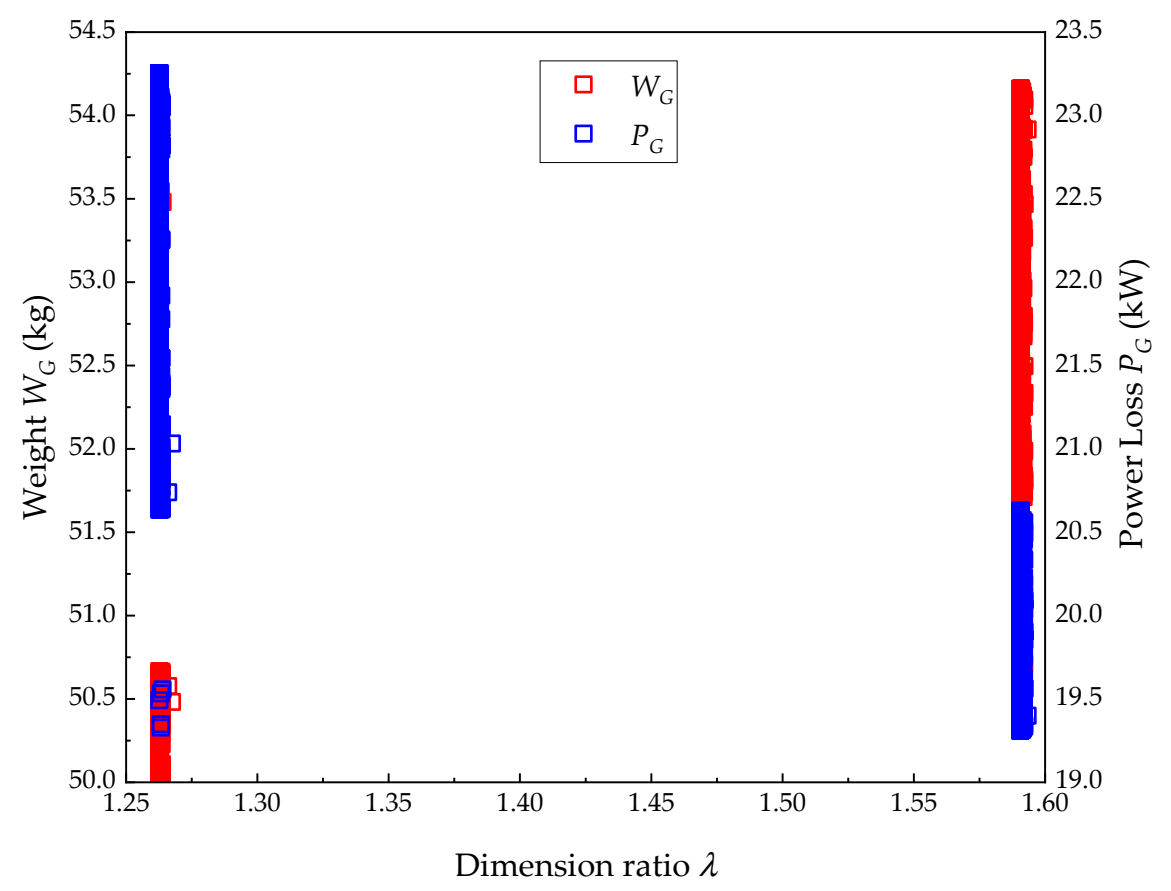

Figure 10. Pareto results with the dimension ratio $\lambda$.

Figure 11 illustrates the data distributions of component operating points in preferred optimal schemes. To achieve the best design results, values of pole and rotor yoke inductions $\left(B_{p}\right.$ and $\left.B_{r y}\right)$ should be at the upper level, whereas those of stator slot and yoke inductions ( $B_{s t}$ and $\left.B_{s y}\right)$ should be at the lower level, as shown in Figure 11a-d. It indicates that inductions $B_{p}, B_{r y}, B_{s t}$, and $B_{s y}$ have few influences on the upper-level designs if the machine is well integrated. Therefore, they are local design variables, which can be ignored in the system-level design. In contrast, values of the current densities are spread relatively evenly in the full ranges given in Table 1, compared with other variables; see Figure 11e-f. The maximum changes of weight and power loss resulting from variations of the 
armature winding current density $J_{a}$ and the excitation winding current density $J_{f}$ are $6.88 \%$ and $1.91 \%$, respectively, which suggests that current densities can also be ignored. In addition, it is notable that the models are convex functions of the current densities, as few data exist for the current densities of the armature and excitation windings in the ranges of 7.2-7.6 and $5-6.5 \mathrm{~A} / \mathrm{mm}^{2}$, respectively.

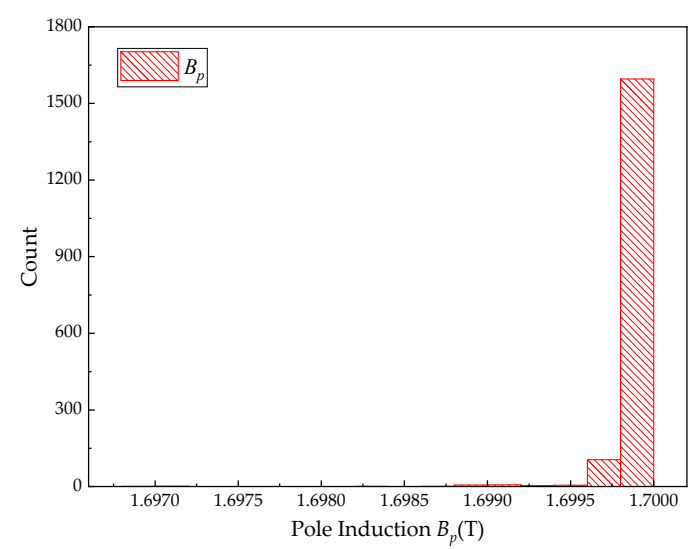

(a)

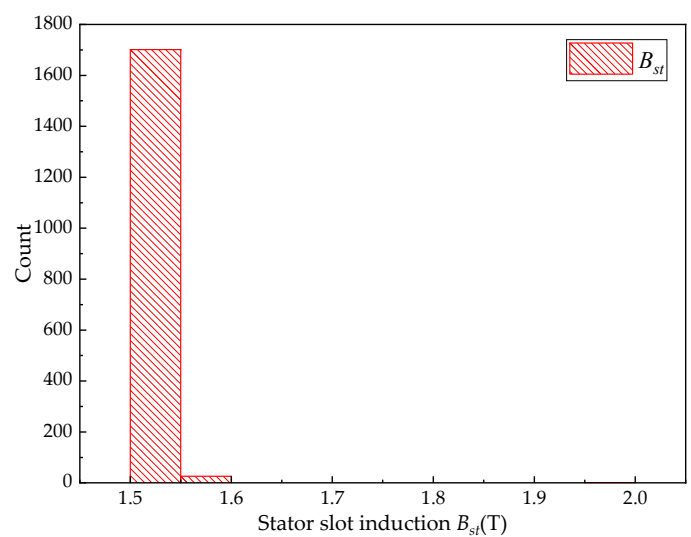

(c)

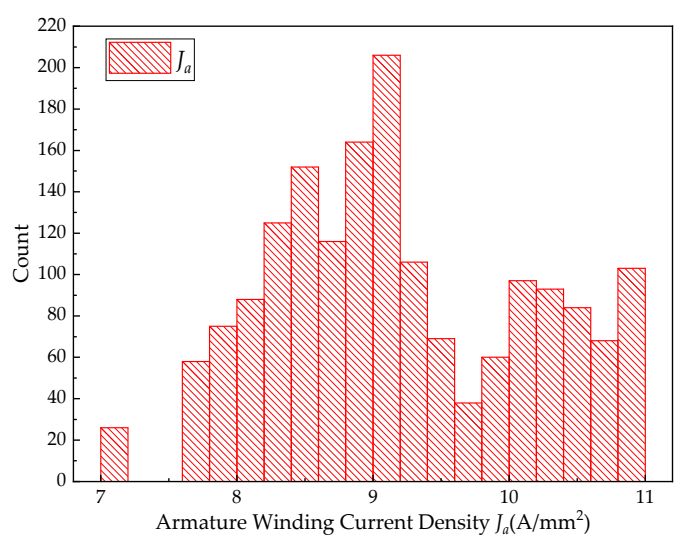

(e)

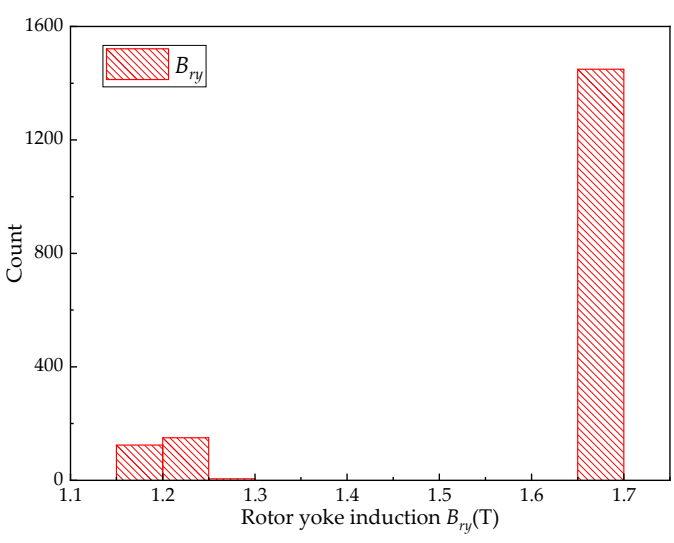

(b)

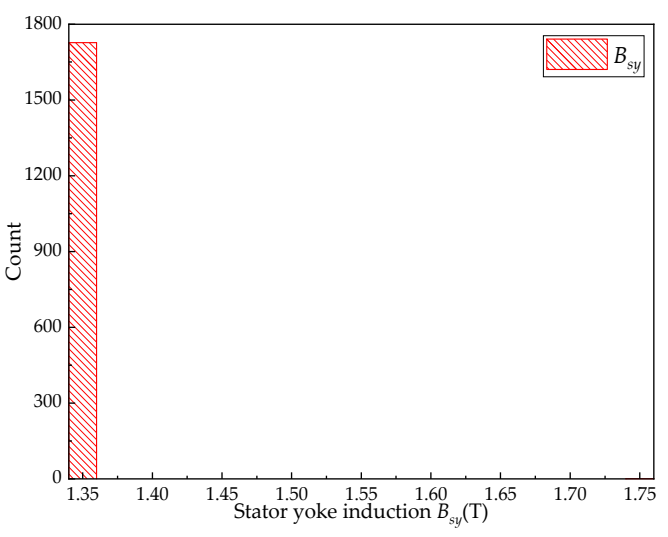

(d)

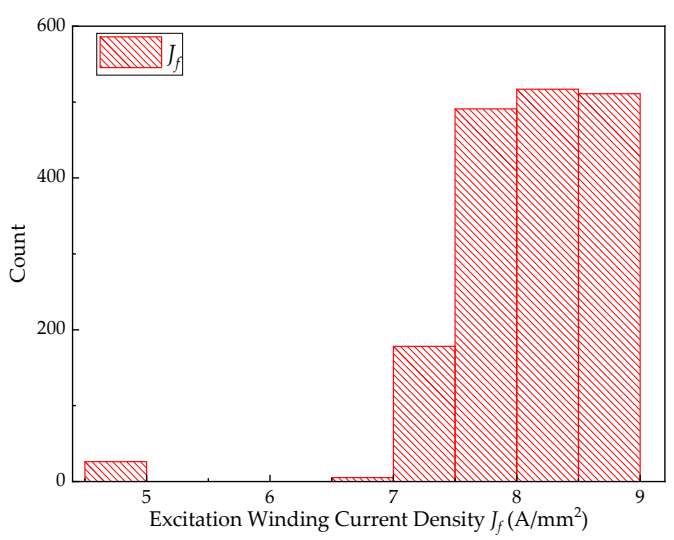

(f)

Figure 11. Data distribution of component operating points to be optimized in the preferred Pareto set: (a) pole induction $B_{p} ;(\mathbf{b})$ rotor yoke induction $B_{r y} ;(\mathbf{c})$ stator slot induction $B_{s t} ;$ (d) slot yoke induction $B_{s y} ;(\mathbf{e})$ armature winding current density $J_{a} ;(\mathbf{f})$ excitation winding current density $J_{f}$. 
In conclusion, among the undetermined design variables, $\lambda$ has a relatively large influence on the weight and power loss, but can be ignored with a high-weighted coefficient of power loss considered in the design and evaluation, whereas component operating points $\left(B_{p}, B_{r y}, B_{s t}, B_{s y}, J_{a}\right.$, and $\left.J_{f}\right)$ can also be reduced due to the optimal design results with few variations. Therefore, the dimensionality reduction is proved conclusive.

\subsubsection{Simplified Model Analysis}

Compared with the actual solution, the errors of the simplified weight and power loss models are acceptably low, at $1.06 \%$ and $-2.03 \%$, respectively. As the surrogate model is a 'black-box' model, the established models are of low complexity. Compared with the weight and power loss of simplified models, the relative variation ranges of the optimal solutions obtained from the multi-physics model on the right of M1 are $-3.574-3.067 \%$ and $-0.526-5.299 \%$ respectively, which indicates that the models output almost the same results. In summary, they are proved to be accurate, simple, and consistent.

In conclusion, the simplification is proved to be reliable, and the multi-physics model and simplified models are of high accuracy and consistency.

\subsection{Comparisons}

In order to demonstrate the superior performance of the proposed modeling method in an application of aircraft power systems, the proposed modeling method is compared with the baseline model of the permanent magnet synchronous motor (PMSG) in [29] and the SL-based model of PMSM in [30].

As the design of the whole aircraft power system is a multi-disciplinary problem resulting from strongly coupled parameters, to achieve the maximum benefit, global optimization solutions are the targets of designers, which indicates that all design variables should be considered at the system level. However, the large amount of on-board equipment leads system-level variables to a high-dimensional design space. Therefore, in addition to the precision, the dimensionality of design space is selected as the compared index to evaluate the performance of the model in system-level applications. As the calculation speed cannot be assessed in the same condition, the complexities of models are compared simply in theory.

\subsubsection{Comparison with the Baseline Model}

To model the PMSG, we replace the electrical excitation modules (silicon steel pole modules and excitation winding modules) by NdFeB magnet poles, and obtain the simplified PMSG model. Design variables are deduced and obtained combining parameters in [29] and equations in this paper, e.g., rated power $P=7.753 \mathrm{~kW}$, pole pairs $p=3$, rotational speed $n=735.2 \mathrm{r} / \mathrm{min}$, electric loading $A=3.69 \times 10^{4} \mathrm{~A} / \mathrm{m}$, air-gap flux density $B_{\delta}=0.856 \mathrm{~T}$, and pitch ratio $\beta=2 / 3$.

According to the improvements of the model mentioned in Section 3.8, the influences of the structure change on the accuracy can be ignored due to the modular modeling structure, whereas those of the integration of design codes and extended input variables remain to be considered, e.g., impacts of different empirical formulas for the calculation of air-gap lengths. As detailed information is not given, only the total model behaviors are compared; see Table 4 . It can be noted that, compared with the prototype, there is a remarkable agreement between the results of the proposed simplified model and the corresponding models in [29].

The input number of the weight model in [29] is eight, whereas that of the simplified weight model in this paper is six, which indicates that a lower dimensionality of design space is needed to be considered in the system-level studies using the proposed method, leading to easier designs. Therefore, with the help of the proposed model, lower computational cost and faster development with considerable accuracy can be achieved.

Moreover, the 'black-box' characteristic of the proposed model indicates that its calculation speed is clearly superior to that of the baseline model. 
Table 4. Model results and error comparisons.

\begin{tabular}{|c|c|c|c|c|c|c|c|}
\hline Parameters & Prototype in [29] & FEM in [29] & $\begin{array}{l}\text { Multi-Physics } \\
\text { Model in [29] }\end{array}$ & $\begin{array}{c}\text { Proposed } \\
\text { Simplified Model }\end{array}$ & $\begin{array}{l}\text { Errors of FEM } \\
\text { in }[29] / \%\end{array}$ & $\begin{array}{c}\text { Errors of } \\
\text { Multi-Physics } \\
\text { Model in [29]/\% }\end{array}$ & $\begin{array}{c}\text { Errors of Proposed } \\
\text { Models/\% }\end{array}$ \\
\hline$R_{a}(\Omega)$ & 0.14 & 0.14 & 0.13 & 0.1348 & 0 & 7.14 & 3.71 \\
\hline$L_{a}(\mathrm{mH})$ & 1.42 & 1.4 & 1.37 & 1.34 & 1.41 & 3.52 & 5.63 \\
\hline$\delta(\mathrm{mm})$ & 1.8 & 1.17 & 1.17 & 1.853 & 35 & 35 & 2.94 \\
\hline lef $(\mathrm{mm})$ & 48.5 & 48.6 & 48.6 & 47.4 & 0.21 & 0.21 & 2.27 \\
\hline$D_{a}(\mathrm{~mm})$ & 141 & - & - & 137.6 & - & - & 2.41 \\
\hline$D_{s y}(\mathrm{~mm})$ & 211.3 & - & - & 193.6 & - & - & 8.38 \\
\hline$W_{G}(\mathrm{~kg})$ & 9.8 & - & - & 9.04 & - & - & 7.76 \\
\hline
\end{tabular}

\subsubsection{Comparison with the SL-Based Model}

To model the PMSM, we change the radial positions of the pole module and slot/armature winding modules inside the PMSG model. As products in [50,51] are of the same series, one reference is selected to identify the variables of the built model, e.g., the electric loading $A=5.62 \times 10^{4} \mathrm{~A} / \mathrm{m}$, air-gap flux density $B_{\delta}=0.85 \mathrm{~T}$, and pitch ratio $\beta=2 / 3$; these values can be used for the whole series.

The relationship between the rated torque and weight is demonstrated in Figure 12. It can be concluded that, although the actual weight includes external accessories besides the active mass, e.g., the resolver and cable, both models achieve results with relatively high accuracy.

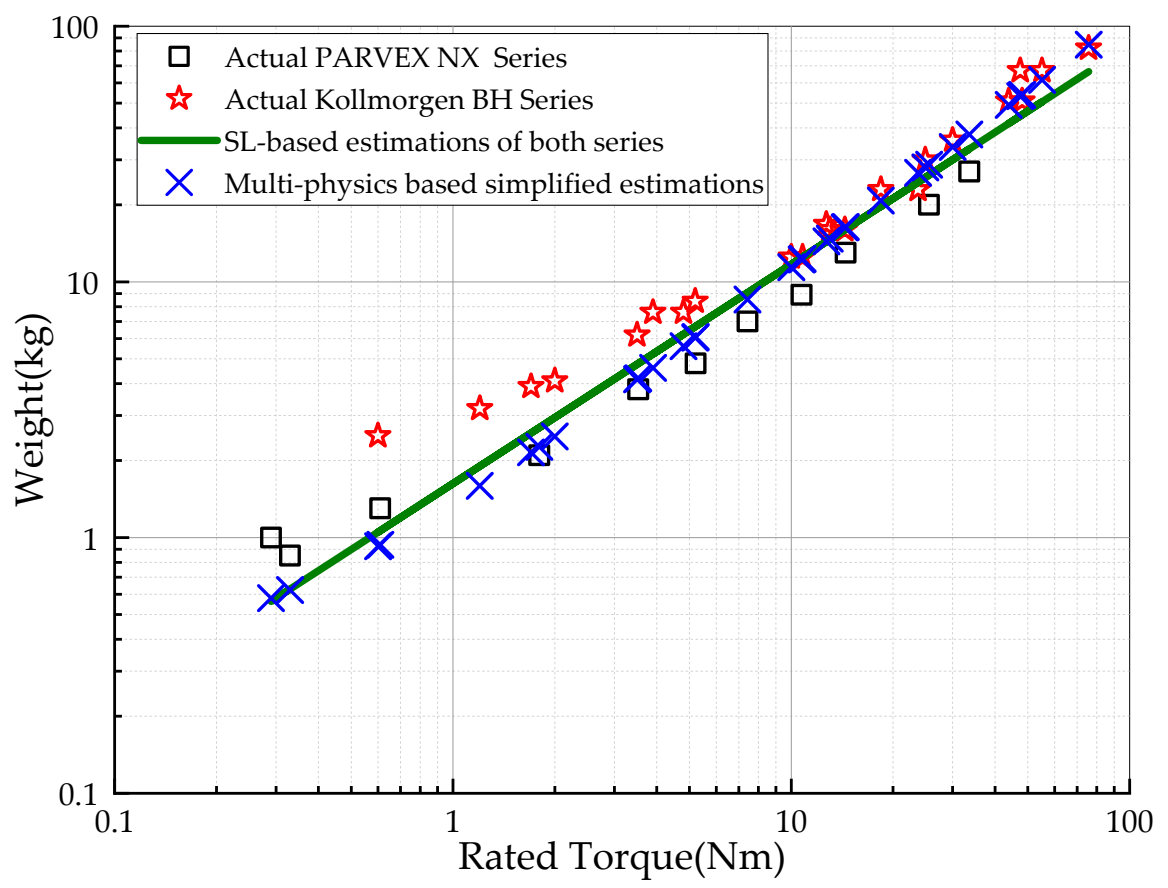

Figure 12. Weights of servo motor series of different models.

The SL-based method requires three inputs for the weight calculation, i.e., rated torque and weight of the reference machine, and rated torque of the machine to be calculated, which is less than the input number of our model. The power loss estimation requires 10 inputs, i.e., values of power and efficiency of the reference in two different conditions of loads (torque and speed) to calculate the coefficients of the Joule loss and iron loss, and torque and speed of the machine to be calculated. The total required input number of the SL-based method is greater than that of the proposed model.

The SL-based model is comprised of several simple polynomials and some data, whereas the proposed model is a relatively complex data network. Therefore, although they have a similar calculation speed, the occupied memory of the proposed model is higher than that of the SL-based model in the optimization process, which can be ignored compared with the generated solutions. 
Moreover, the SL-based model cannot reflect the couplings of multi-physics, which cannot satisfy the study requirements of transport electrification.

Therefore, compared with other models used for the integrated design of aircraft power systems, the proposed method is demonstrated to be of similar accuracy, lower computational cost, and faster calculation speed, and promises to be a powerful tool in the multi-level design of aircraft power systems.

\section{Conclusions}

The objective of this paper was to develop an efficient modeling tool for machine design tasks in different design phases of aircraft power systems. For this purpose, the paper improved the existing multi-physics model and proposes a novel simplification process based on MDO. The major features of the proposed method are:

1. The proposed modular modeling structure of the multi-physics model divides the machine into several replaceable sub-models, enabling the evaluation of different machine types by simply changing sub-models and their integration forms. Design codes are embedded in the model to reduce professional requirements for system integrators and to realize the combination of the principle and practical technologies. Moreover, this modeling method can also be applied to other equipment in the aircraft power system and enables efficient and quick designs with optimization algorithms.

2. With the knowledge analysis of the improved multi-physics model, sensitive variables are selected from the multi-physics model as the inputs of the simplified models. The similar results of two-level models indicate that the nesting designs of different levels can be achieved with consistent results. Therefore, models of different levels are associated and consistent in aspects of interface and results, and the proposed modeling framework offers a systematic evaluation tool for designs of different levels.

3. Compared with existing models for aircraft power system integration, the proposed simplified models of electrical machines have the advantages of lower-dimensional design space and black-box characteristics without reducing the precision. Both fast and accurate designs can be achieved by the method.

Future studies will focus on the simulation-based DOE to extract more detailed performance of machines for holistic evaluations. In addition, power allocations of multiple energy systems, the optimal operating voltage level of power systems, and couplings among More-electric systems (i.e., the gearbox and output frequency, fuel pumps, and cooling, etc.) are planned to be studied for future applications.

Author Contributions: Conceptualization, Z.D. and L.W.; formal analysis, Z.D., L.W., and S.Y.; funding acquisition, L.W.; investigation, Z.D. and L.M. (Ling Mao); methodology, Z.D. and L.W.; project administration, S.Y.; resources, Z.D.; supervision, L.W.; validation, Z.D. and L.M. (Lexuan Meng); visualization, S.Y.; writing-original draft, Z.D.; Writing-review and editing, Z.D., L.W., L.M. (Lexuan Meng) and L.M. (Ling Mao).

Funding: This research was funded by the National Natural Science Foundation of China, grant number 51877102. Conflicts of Interest: The authors declare no conflict of interest.

\section{References}

1. Riu, D.; Sautreuil, M.; Retière, N.; Sename, O. Control and design of DC grids for robust integration of electrical devices. Application to aircraft power systems. Int. J. Electr. Power Energy Syst. 2014, 58, 181-189. [CrossRef]

2. Berton, J.J.; Kim, H.D.; Singh, R.; Tong, M.T.; Haller, W.J.; Felder, J.L. Turboelectric distributed propulsion benefits on the N3-X vehicle. Aircr. Eng. Aerosp. Technol. 2014, 86, 558-561. [CrossRef]

3. Mai, T.; Jadun, P.; Logan, J.; McMillan, C.; Muratori, M.; Steinberg, D.; Vimmerstedt, L.; Jones, R.; Haley, B.; Nelson, B. Electrification Futures Study: Scenarios of Electric Technology Adoption and Power Consumption for 
the United States; Tech. Rep. NREL/TP-6A20-71500; National Renewable Energy Laboratory: Golden, CO, USA, 2018.

4. Cao, W.; Mecrow, B.C.; Atkinson, G.J.; Bennett, W.; Atkinson, D.J. Overview of electric motor technologies used for MEA. IEEE Trans. Ind. Electron. 2012, 59, 3523-3531. [CrossRef]

5. Sadey, D.J.; Csank, J.; Hanlon, P.A.; Jansen, R.H. A generalized power system architecture sizing and analysis framework. In Proceedings of the 2018 AIAA Joint Propulsion Conference, Cincinnati, OH, USA, 1-11 July 2018.

6. Hanlon, P.; Thomas, G.L.; Csank, J.; Sadey, D.J. A tool for modeling and analysis of electrified aircraft power systems. In Proceedings of the AIAA Propulsion an Energy 2019 Forum, Indianapolis, IN, USA, 19-22 August 2019.

7. Csank, J.; Sadey, D.J.; Lavelle, T.M.; Garcia, J.; Bergeson, J. Electrical power system sizing within the numerical propulsion system simulation. In Proceedings of the AIAA Propulsion an Energy 2019 Forum, Indianapolis, IN, USA, 19-22 August 2019.

8. International Council on Systems Engineering. Systems Engineering Handbook: A Guide for System Life Cycle Processes and Activities, 4th ed.; John Wiley and Sons Inc.: San Diego, CA, USA, 2012; ISBN 9781118999400.

9. Kevin, A.R.; Stephen, E.; Russell, P.; Dimitri, M. Methodologies for modeling and simulation in model-based systems engineering tools. In Proceedings of the AIAA SPACE 2016, Long Beach, CA, USA, 13-16 September 2016. [CrossRef]

10. Bals, J.; Hofer, G.; Pfeiffer, A.; Schallert, C. Virtual Iron Bird-a Multidisciplinary Modelling and Simulation Plat-Form for New Aircraft System Architectures. In Deutscher Luft-Und Raumfahrkongress; Friedrichshafen, Ed.; German Society for Aeronautics and Astronautics: Bonn, Germany, 2005; pp. 1-9.

11. Roboam, X.; Sareni, B.; de Andrade, A. More electricity in the air: Toward optimized electrical networks embedded in more-electrical aircraft. IEEE Ind. Electron. Mag. 2012, 6, 6-17. [CrossRef]

12. de Andrade, A.; Lesage, A.; Sareni, B.; Meynard, T.; Roboam, X.; Ruelland, R.; Couderc, M. Integrated optimal design for power systems of more electrical aircraft. In Proceedings of the International Conference Proceedings on More Electric Aircraft, Bordeaux, France, 20-21 November 2012; pp. 1-8.

13. Ounis, H.; Sareni, B.; Roboam, X.; de Andrade, A. Multi-level integrated optimal design for power systems of more electric aircraft. Math. Comput. Simul. 2015, 130, 223-235. [CrossRef]

14. Budinger, M.; Reysset, A.; Halabi, T.E.; Vasiliu, C.; Maré, J.C. Optimal preliminary design of electromechanical actuators. Proc. Inst. Mech. Eng. Part G J. Aerosp. Eng. 2012, 226, 243-259. [CrossRef]

15. Reysset, A. Preliminary Design of Electromechanical Actuators-Development of Tools Dedicated to Technical Specification and Optimal Sizing Sequence Conditioning. Ph. D. Thesis, Department Mechanical Engineering, Université de Toulouse, Toulouse, France, 3 February 2015.

16. Wen, B.; Zhang, X.; Effah, F. Integrated design by optimization of electrical power systems for more electric aircraft. In Proceedings of the MEA 2015, Toulouse, France, 3-5 February 2015.

17. Fu, J.; Mare, J.C.; Yu, L.; Fu, Y. Multi-level virtual prototyping of electromechanical actuation system for more electric aircraft. Chin. J. Aeronaut. 2018, 31, 892-913. [CrossRef]

18. Garriga, A.G.; Govindaraju, P.; Ponnusamy, S.S.; Cimmino, N.; Mainini, L. A modelling framework to support power architecture trade-off studies for more-electric aircraft. Transp. Res. Procedia 2018, 29, 146-156. [CrossRef]

19. Xue, L.; Wu, S.; Xu, Y.; Ma, D. A simulation-based multi-objective optimization design method for pump-driven electro-hydrostatic actuators. Processes 2019, 7, 274. [CrossRef]

20. Bazzo, T.D.; Kölzer, J.F.; Carlson, R.; Wurtz, F.; Gerbaud, L. Multiphysics design optimization of a permanent magnet synchronous generator. IEEE Trans. Ind. Electron. 2017, 64, 9815-9823. [CrossRef]

21. Ostovic, V. Dynamics of Saturated Electric Machines; Springer: New York, NY, USA, 1989; pp. 5-138, ISBN 978-1-4613-8935-4.

22. Keysan, O.; Mcdonald, A.; Mueller, M. A Direct Drive Permanent Magnet Generator Design for a Tidal Current Turbine (SeaGen); IEMDC: Niagara Falls, ON, Canada; IEEE: New York, NY, USA, 2011.

23. Ursu, D.; Tutelea, L.; Ionel, D.; Boldea, I. Optimal Design of BLDC Multi-Phase Reluctance Machines (MRM) for Variable Speed Drives; OPTIM \& ACEMP: Brasov, Romania; IEEE: New York, NY, USA, 2017.

24. Bendib, M.; Taib, M.; Mekias, A. Design of Axial Flux Pm Machine for Flywheel Energy Storage System; ICWEAA: Algiers, Algeria; IEEE: New York, NY, USA, 2018. 
25. Staubach, C.; Wulff, J.; Jenau, F. Particle Swarm Based Simplex Optimization Implemented in a Nonlinear, Multiple-Coupled Finite-Element-Model for Stress Grading in Generator end Windings; OPTIM: Brasov, Romania; IEEE: New York, NY, USA, 2012.

26. Stipetic, S.; Miebach, W.; Zarko, D. Optimization in Design of Electric Machines: Methodology and Workflow; ACEMP, OPTIM \& ELECTROMOTION: Side, Turkey; IEEE: New York, NY, USA, 2015.

27. Saygin, A.; Aksöz, A. Design Optimization for Minimizing Cogging Torque in Axial Flux Permanent Magnet Machines; OPTIM \& ACEMP: Brasov, Romania; IEEE: New York, NY, USA, 2017.

28. Vivier, S.; Friedrich, G. Comparison between single-model and multimodel optimization methods for multiphysical design of electrical machines. IEEE Trans. Ind. Appl. 2018, 54, 1379-1389. [CrossRef]

29. Sareni, B.; Abdelli, A.; Roboam, X.; Tran, D.H. Model simplification and optimization of a passive wind turbine generator. Renew. Energy 2009, 34, 2640-2650. [CrossRef]

30. Budinger, M.; Liscouët, J.; Hospital, F.; Maré, J.C. Estimation models for the preliminary design of electro-mechanical actuators. Proc. Inst. Mech. Eng. Part G J. Aerosp. Eng. 2012, 226, 243-259. [CrossRef]

31. Mohamed, F.A.; Koivo, H.N. System modeling and online optimal management of microgrid using multi-objective optimization. In Proceedings of the International Conference on Clean Electrical Power, Capri, Itlay, 21-23 May 2007; IEEE: New York, NY, USA, 2007.

32. McCarthy, K.; McCarthy, P.; Wu, N.; Alleyne, A.; Koeln, J.; Patnaik, S.; Emo, S.; Cory, J. Model accuracy of variable fidelity vapor cycle system simulations. Sae Tech. Pap. 2014, 2014, 1-13. [CrossRef]

33. Wang, L.; Dai, Z.H.; Yang, S.S.; Mao, L.; Yan, Y.G. Review of intelligent design of electrified aircraft power system. Acta Aeronaut. Et Astronaut. Sin. 2019, 40, 522405. [CrossRef]

34. Lei, G.; Liu, C.C.; Guo, Y.G.; Zhu, J.G. Robust multidisciplinary design optimization of PM machines with soft magnetic composite cores for batch production. IEEE Trans. Magn. 2015, 52, 1-4. [CrossRef]

35. Levi, E. Polyphase Motors: A Direct Approach to Their Design; Wiley: New York, NY, USA, 1984; pp. $131-171$.

36. Silva, R.C.; Li, M.; Rahman, T.; Lowther, D.A. Surrogate-based MOEA/D for electric motor design with scarce function evaluations. IEEE Trans. Magn. 2017, 53, 7400704. [CrossRef]

37. Bramerdorfer, G.; Zăvoianu, A.C. Surrogate-based multi-objective optimization of electrical machine designs facilitating tolerance analysis. IEEE Trans. Magn. 2017, 53, 8107611. [CrossRef]

38. Dai, W.J.; Zhang, J.M. Machine Design; Tsinghua University Press: Beijing, China, 2010; pp. 1-2, ISBN ECCE 2015.

39. International Electrotechnical Commission. Laminations for transformers and inductors-Part 1: Mechanical and electrical characteristics. In IEC 60740-1:2005; International Electrotechnical Commission: Geneva, Switzerland, 2005.

40. SAE AE-8A System Installation Committee. Wiring Aerospace Vehicle, SAE AS50881F; SAE International: Warrendale, PA, USA, 2015.

41. International Electrotechnical Commission. Basic Dimensions of Winding Wires, IEC 60182:1984; International Electrotechnical Commission: Geneva, Switzerland, 1984.

42. Tran, D.H.; Sareni, B.; Roboam, X.; Espanet, C. Integrated optimal design of a passive wind turbine system: An experimental validation. IEEE Trans. Sustain. Energy 2010, 1, 48-56. [CrossRef]

43. Lim, D.H.; Kim, S.C. Thermal performance of oil spray cooling system for in-wheel motor in electric vehicles. Appl. Therm. Eng. 2014, 63, 577-587. [CrossRef]

44. Bazzo, T.; Kolzer, J.F.; Carlson, R.; Wurtz, F.; Gerbaud, L. Optimum Design of a High-Efficiency Direct-Drive PMSG; ECCE: Montreal, QC, Canada; IEEE: New York, NY, USA, 2015.

45. Saari, J. Thermal Analysis of High-Speed Induction Machines. Ph. D. Thesis, Department EE, Helsinki University of Technology, Helsinki, Finland, 30 June 1998.

46. McKay, M.D.; Beckman, R.J.; Conover, W.J. A comparison of three methods for selecting values of input variables in the analysis of output from a computer code. Technometrics 1979, 21, 239-245. [CrossRef]

47. Iman, R.L.; Helton, J.C.; Campbell, J.E. An approach to sensitivity analysis of computer models, Part 1. Introduction, input variable selection and preliminary variable assessment. J. Qual. Technol. 1981, 13, 174-183. [CrossRef]

48. Jin, R.; Chen, W.; Sudjianto, A. An efficient algorithm for constructing optimal design of computer experiments. J. Stat. Plan. Inference 2004, 134, 268-287. [CrossRef]

49. Hamilton Sundstrand Co. Component Maintenance Manual with Illustrated Parts List-Auxiliary Power Unit Start Generator; Hamilton Sundstrand Co.: Windsor Locks, CT, USA, 2015; Revision 18. 
50. Parker, H. Servomotors NX Series Technical Manual, Edition 03/2017 ed; Parker Hannifin: Longvic Cedex, France, 2017.

51. Kollmorgen. Synchronous Servomotors Series GOLDLINE ${ }^{\mathrm{TM}}$ BH, Technical Description, Installation, Commissioning, Edition 07/2000 ed; Kollmorgen: Radford, VA, USA, 2000. 